\title{
The stilbene profile in edible berries
}

\author{
Alfred Błaszczyk $(\mathbb{D} \cdot$ Sylwia Sady $\cdot$ Maria Sielicka
}

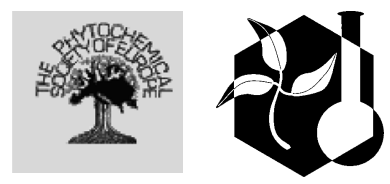

Received: 6 November 2017/Accepted: 7 June 2018/Published online: 15 June 2018

(C) The Author(s) 2018

\begin{abstract}
Edible berries are becoming increasingly popular to consume in fresh, dried, frozen or processed forms due to their high content and wide diversity of bioactive compounds with considerable health benefits. Among the wide variety of phytochemicals found in berries are stilbenes, which demonstrate a broad range of biological and pharmacological activities. Their content depends on many factors, including the cultivar, ripening stage, climatic conditions, agronomic management, storage conditions and postharvest management. However, the application of various abiotic and biotic external stimuli could be a strategy for increasing the production of stilbenes in edible berries. To date, several different elicitors, as inducers of plant secondary metabolite stilbenes, have been applied in different studies. This review focuses on the isolation and identification of stilbenes from edible berries and presents the influence of different external stimuli on their profile in grapes.
\end{abstract}

Keywords Stilbenes · Edible berries · External stimuli

A. Błaszczyk $(\bowtie) \cdot$ S. Sady $\cdot$ M. Sielicka

Faculty of Commodity Science, Poznań University of

Economics and Business, al. Niepodległości 10,

61-875 Poznań, Poland

e-mail: alfred.blaszczyk@ue.poznan.pl

\section{Introduction}

In recent years, edible berries have attracted much interest due to their high content and wide diversity of bioactive compounds with potential health benefits (Zhao 2007; Seeram 2012; Jimenez-Garcia et al. 2013; Nile and Park 2014). Berry fruits are widely consumed in fresh, dried, frozen forms or as processed products, including canned fruits, beverages, jams and yogurts. The most consumed berries are red raspberries (Rubus idaeus), strawberries (Fragaria $x$ ananassa), blackberries (Rubus spp.), blueberries (Vaccinium corymbosum), black currants (Ribes nigrum), red currants (Ribes rubrum), chokeberries (Aronia melanocarpa), cranberries (Vaccinium macrocarpon), grapevines (Vitis vinifera L. and other Vitis species), bilberries (Vaccinium myrtillus L.), deerberries (Vaccinium stamineum L.), cowberries (Vaccinium vitis-idaea L.), passion fruits (Passiflora edulis) and tomatoes (Lycopersicon esculentum Mill.).

Among the wide variety of phytochemicals found in berries are stilbenes. These molecules occur within a limited group of plant families, which have the gene encoding the enzyme stilbene synthase (STS, EC 2.3.1.95). Biosynthesis occurs via the phenylalanine pathway, where phenylalanine ammonia lyase $(P A L)$, cinnamate-4-hydroxylase $(\mathrm{C} 4 \mathrm{H})$, coumaroyl-CoA ligase (4CL) and STS play a core role in the synthesis. (Fig. 1) A transcriptional factor, Myb14, has been found to regulate the expression of STS (Holl et al. 
Fig. 1 Early stages of stilbene biosynthesis. $P A L$ phenylalanine ammonia lyase, C4H cinnamate-4hydroxylase, 4CL coumaroyl-CoA ligase, CHS chalcone synthase, STS stilbene synthase

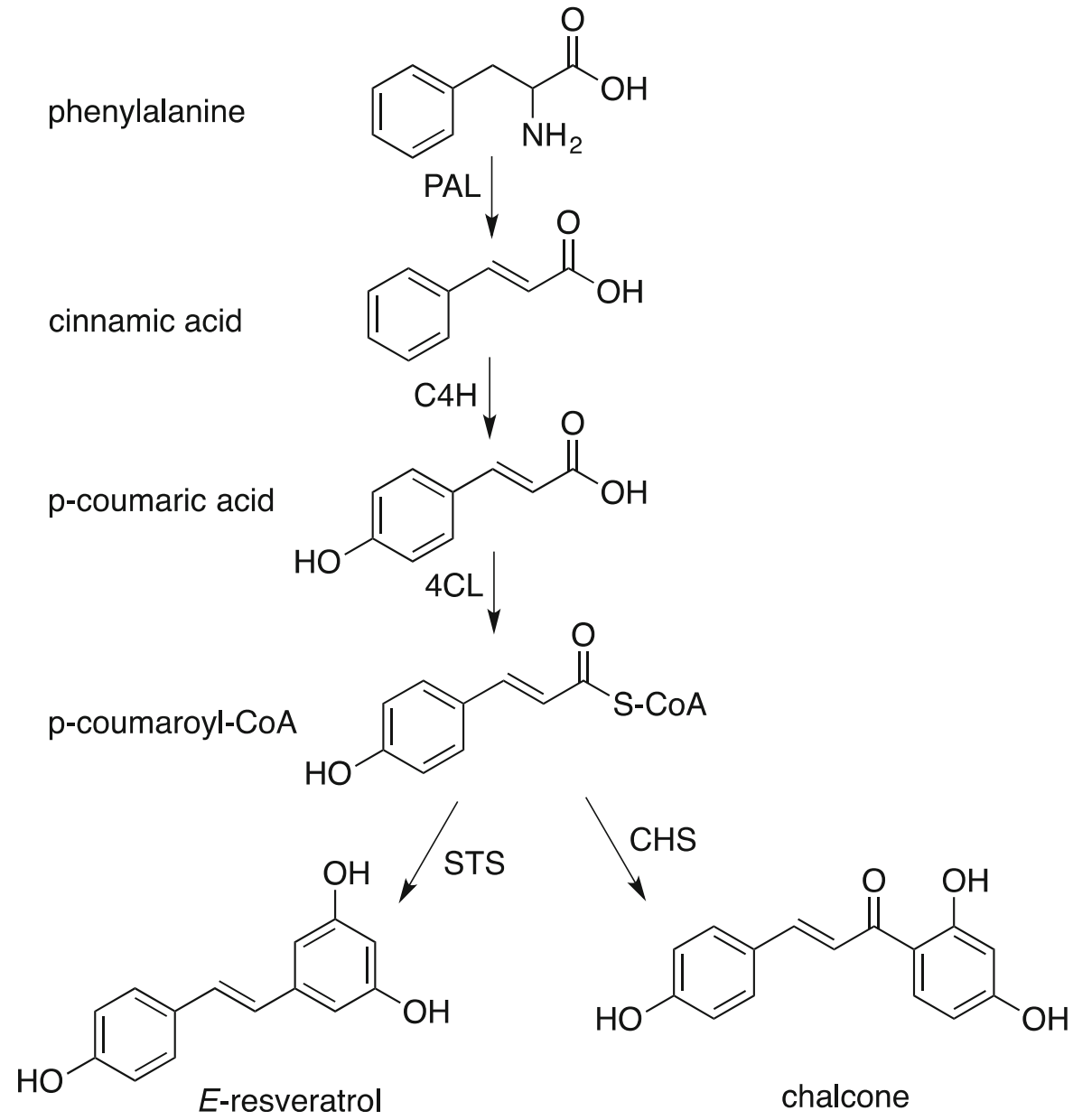

2013). Chalcone isomerase $(\mathrm{CHI})$ is responsible for the conversion of chalcone to flavanones. On the other hand, resveratrol O-methyltransferase (ROMT) is involved in the methylation of resveratrol. Stilbenes are biosynthesized and accumulate into lipid vesicles in the cytoplasm. Their content can be determined by many factors, including the cultivar, ripening stage, climatic conditions, soil type, agronomic management, storage conditions and postharvest management (Dixon and Paiva 1995; Castrejon et al. 2008). Due to the potential health benefits, stilbenes in edible fruits are of high interest. These compounds have demonstrated a wide range of biological and pharmacological activities, including anti-tumoural (Bai et al. 2010; Tsai et al. 2017), anti-viral (Nguyen et al. 2011), antiinflammatory (Zhang et al. 2010), anti-atherogenic (Ramprasath and Jones 2010), anti-aging (Kasiotis et al. 2013) and neuroprotective (Lin and Yao 2006) effects.
This review focuses on stilbenes as a specific class of non-flavonoid phenolic compounds present in edible berries. The aim of this review is to summarize the isolation and identification methods applied for stilbenes present in edible berries as well as the influence of external stimuli on the quantitative and qualitative composition of stilbenes in edible berries.

\section{Molecular structures of stilbenes found in berry fruits}

The stilbene structure is characterized by two aromatic rings linked by a double bond, of which the $E$ isomer is the most common configuration. They can be found in berry fruits as monomers, dimers and more complex oligomers (Figs. 2, 3, 4, 5, 6,7). According to a current paper, the most widely found monomeric stilbenes in berry fruits are $E$-resveratrol (1) and $E$-piceid (3) 


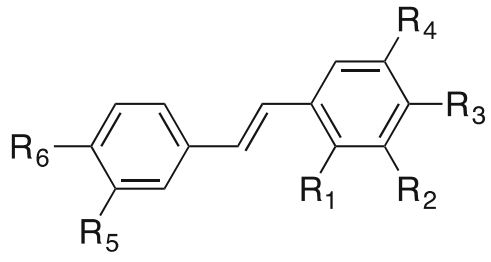

$1 \mathrm{R}_{1}, \mathrm{R}_{3}, \mathrm{R}_{5}=\mathrm{H}, \mathrm{R}_{2}, \mathrm{R}_{4}, \mathrm{R}_{6}=\mathrm{OH}$

$2 \mathrm{R}_{1}, \mathrm{R}_{3}, \mathrm{R}_{5}=\mathrm{H}, \mathrm{R}_{2}, \mathrm{R}_{4}=\mathrm{OCH}_{3}, \mathrm{R}_{6}=\mathrm{OH}$

$3 \mathrm{R}_{1}, \mathrm{R}_{3}, \mathrm{R}_{5}=\mathrm{H}, \mathrm{R}_{2}, \mathrm{R}_{6}=\mathrm{OH}, \mathrm{R}_{4}=\mathrm{OGlC}$

$4 \mathrm{R}_{1}, \mathrm{R}_{3}=\mathrm{H}, \mathrm{R}_{2}, \mathrm{R}_{4}, \mathrm{R}_{5}, \mathrm{R}_{6}=\mathrm{OH}$

$5 \mathrm{R}_{1}, \mathrm{R}_{3}=\mathrm{H}, \mathrm{R}_{4}, \mathrm{R}_{5}, \mathrm{R}_{6}=\mathrm{OH}, \mathrm{R}_{2}=$ OGlc

$6 \mathrm{R}_{1}, \mathrm{R}_{3}=\mathrm{H}, \mathrm{R}_{4}, \mathrm{R}_{6}=\mathrm{OH}, \mathrm{R}_{5}=\mathrm{OCH}_{3}, \mathrm{R}_{2}=\mathrm{OGlc}$

Fig. 2 Molecular structures of $E$-stilbene monomers isolated from edible berries: 1: $E$-resveratrol $\left(3,4^{\prime}, 5\right.$-trihydroxy- $E$ stilbene), 2: 3,5-O-dimethyl- $E$-resveratrol ( $E$-pterostilbene), 3 : $E$-resveratrol-3-O- $\beta$-D-glucopyranoside ( $E$-piceid), 4: 3,3',4,5tetrahydroxy- $E$-stilbene ( $E$-piceatannol), 5: $E$-piceatannol-3-O$\beta$-D-glucopyranoside ( $E$-astringin), 6: isorhapontin

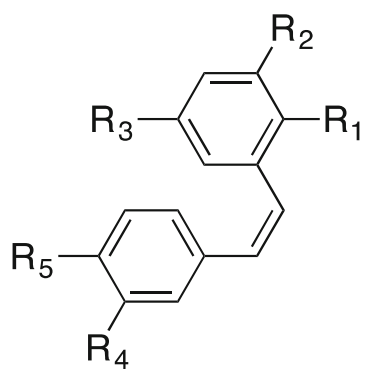

$$
\begin{aligned}
& 7 \mathrm{R}_{1}, \mathrm{R}_{3}, \mathrm{R}_{5}=\mathrm{H}, \mathrm{R}_{2}, \mathrm{R}_{4}, \mathrm{R}_{6}=\mathrm{OH} \\
& \mathbf{8} \mathrm{R}_{1}, \mathrm{R}_{4}=\mathrm{H}, \mathrm{R}_{2}, \mathrm{R}_{5}=\mathrm{OH}, \mathrm{R}_{3}=\mathrm{OGIC} \\
& \text { 9 } \mathrm{R}_{1}=\mathrm{H}, \mathrm{R}_{3}, \mathrm{R}_{4}, \mathrm{R}_{5}=\mathrm{OH}, \mathrm{R}_{2}=\text { OGlc }
\end{aligned}
$$

Fig. 3 Molecular structures of $Z$-stilbene monomers isolated from edible berries: 7: $Z$-resveratrol, 8: $Z$-resveratrol-3-O- $\beta$-Dglucopyranoside (Z-piceid), 9: $Z$-piceatannol-3-O- $\beta$-D-glucopyranoside ( $Z$-astringin)

(Fig. 2). Their $Z$ - and $E$-isomers mainly accumulate in the berry skin during all stages of development (Jeandet et al. 1991). E-isomer plant stilbenes may undergo several types of modifications, such as isomerisation, glycosylation, methoxylation, and oligomerization (Chong et al. 2009). Due to these modifications, different derivatives of stilbenes are formed in edible berries from dimers to hexamers (Figs. 2, 3, 4, 5, 6, 7). In plants, these metabolites generally accumulate in both free and glycosylated forms. Glycosylation of stilbenes could be involved in their storage, transport from the cytoplasm to the apoplasm and protection from peroxidative degradation (Morales et al. 1998). The oligomeric stilbenes are formed due to the oxidative coupling of $E$-resveratrol (1) or other monomeric stilbenes catalysed by peroxidase isoenzymes localized in the vacuole, cell wall and apoplast of grapevine cells (Ros Barcelo et al. 2003).

\section{Isolation and identification of stilbenes in edible berries}

The isolation and identification of stilbenes in edible berry extracts constitutes a complex procedure due to complex composition of the matrices in which their occur, their low concentration and structural complexity. Several strategies have been applied for the isolation and identification of stilbenes in edible berries. An overview of the preparation conditions, analytical methods and stilbene concentrations in accordance with the research objectives are presented in Table 1. In general, the extraction techniques applied in stilbene extraction are classified into two categories: conventional and green techniques. The conventional techniques involve soaking in solvent, which relies on the solubility of stilbenes from edible berries in the solvent at room or elevated temperature. These techniques consume a large volume of solvents and are usually time consuming. In contrast, the green extraction technique applies minimal volumes of solvent and requires a shorter time. It has been used as preparation procedure by Ehala et al. (2005) to isolate $E$-resveratrol from bilberry via microwaveassisted extraction. In conventional extraction techniques of stilbenes from edible berries solid-liquid extraction of lyophilized, air-dried, frozen or fresh samples with different solvents is applied (see Table 1). Unfortunately, there are no studies in which the influence of matrices (lyophilized, air-dried, frozen, fresh) on the presence of stilbenes was examined. From a quantitative point of view, the results obtained by different extraction solvents cannot be compared because solvents of different natures have different extractabilities. Basing on the analysed works (Table 1), the most often used solvent for the extraction process is methanol or the mixtures of methanol with other solvents. In the research conducted by Sun et al. (2006) and Romero et al. (2001) the influence of extraction solvents on the amount of 

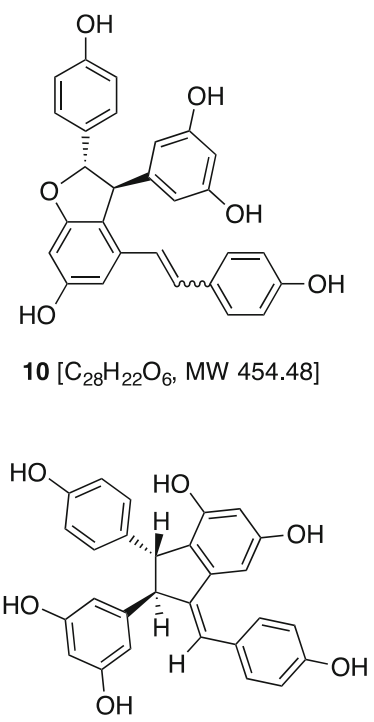

$14\left[\mathrm{C}_{28} \mathrm{H}_{22} \mathrm{O}_{6}, \mathrm{MW} 454.48\right]$

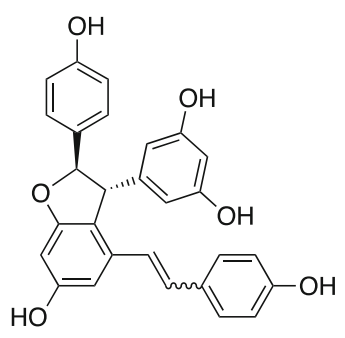

$11\left[\mathrm{C}_{28} \mathrm{H}_{22} \mathrm{O}_{6}, \mathrm{MW} 454.48\right]$

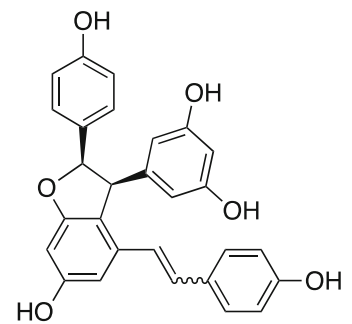

$12\left[\mathrm{C}_{28} \mathrm{H}_{22} \mathrm{O}_{6}, \mathrm{MW} 454.48\right]$

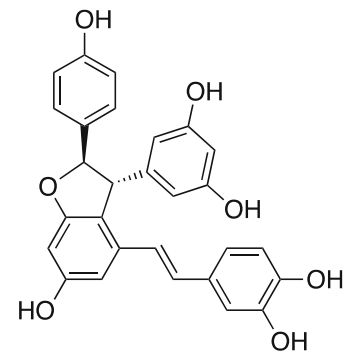

$13\left[\mathrm{C}_{28} \mathrm{H}_{22} \mathrm{O}_{7}\right.$, MW 470.48]<smiles>[R]c1cc(O)c2c(c1)[C@@H]1[C@@H]2[C@H]2[C@H](c3ccc(O)cc3)c3c(O)cc(O)cc3[C@H]2[C@H]1c1ccc(O)cc1</smiles>

15: $\mathrm{R}=\mathrm{OH}\left[\mathrm{C}_{28} \mathrm{H}_{22} \mathrm{O}_{6}, \mathrm{MW} 454.48\right]$ 16: $\mathrm{R}=\mathrm{OGlc}\left[\mathrm{C}_{34} \mathrm{H}_{32} \mathrm{O}_{11}, \mathrm{MW} 616.62\right]$

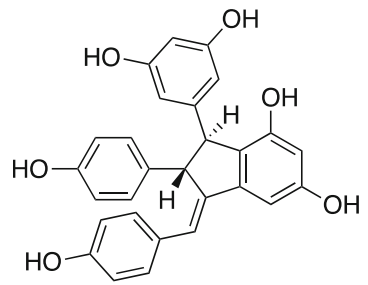

$17\left[\mathrm{C}_{28} \mathrm{H}_{22} \mathrm{O}_{6}\right.$, MW 454.48]<smiles>O=C(c1ccc(O)cc1)[C@H]1c2cc(O)cc(O)c2[C@H](c2cc(O)cc(O)c2)[C@H]1c1cc(O)cc(O)c1</smiles>

$18\left[\mathrm{C}_{28} \mathrm{H}_{22} \mathrm{O}_{8}, \mathrm{MW} 486.48\right]$

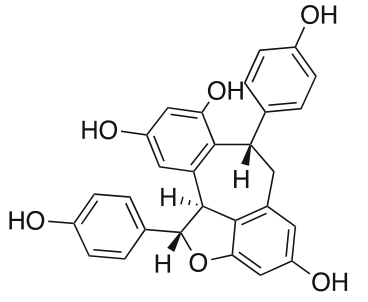

$19\left[\mathrm{C}_{28} \mathrm{H}_{22} \mathrm{O}_{8}, \mathrm{MW} 486.48\right]$
Fig. 4 Molecular structures of stilbene dimers isolated from edible berries: 10: $E$ - and $Z$ - $\varepsilon$-viniferin, 11: $E$ - and $Z$ - $\delta$-viniferin, 12: $E$ - and $Z$ - $\omega$-viniferin, 13: scirpusin $B, 14$ : parthenocissin $A$,

extracted stilbenes was examined. Based on Sun et al. work (Table 1, entry no. 15), methanol acidified with $0.1 \% \mathrm{HCl}$ was the best solvent to extract specific stilbenes from grape skins and seeds. In the research conducted by Romero et al. (2001) the influence of temperature and time of extraction on stilbenes content was examined. The highest extraction of $E$ resveratrol and piceid isomers was observed at $60{ }^{\circ} \mathrm{C}$ for 30 min with $80 \%$ ethanol. Z-Resveratrol was not detected in any conditions assayed. The longer time of extraction at $60{ }^{\circ} \mathrm{C}$, the lower stilbenes content was measured, probably due to their degradation. It is only known that resveratrol and its glycon piceid are stable at $40{ }^{\circ} \mathrm{C}$ in the presence of ambient air (Prokop et al. 2006). However $E$-isomer is unstable in solution when exposed to light and readily isomerizes to the $Z$ form and other degradants (Jensen et al. 2010).
15: pallidol, 16: pallidol-3-O-glucoside, 17: ampelopsin D, 18: caraphenol B, 19: ampelopsin B

Therefore, the preparation procedure of stilbenes isolation should be carried out in the dark due to the light sensitivity of double bond in stilbenes.

Few studies have dealt with the influence of ultrasound on the resveratrol extraction efficiency from grapes (Burin et al. 2014; Babazadeh et al. 2017). The ultrasonication-assisted extraction of resveratrol showed more efficiency than the conventional solvent extraction with $80 \%$ ethanol at $60{ }^{\circ} \mathrm{C}$ for $30 \mathrm{~min}$. The recovery of resveratrol increased by $24-30 \%$ compared with the conventional solvent extraction (Cho et al. 2006).

To decrease background noise in analytical techniques and improve the identification of stilbenes, the purification stage in the preparation procedure is very often applied. Typically, this purification utilizes an additional extraction with other solvent, often EtOAc 

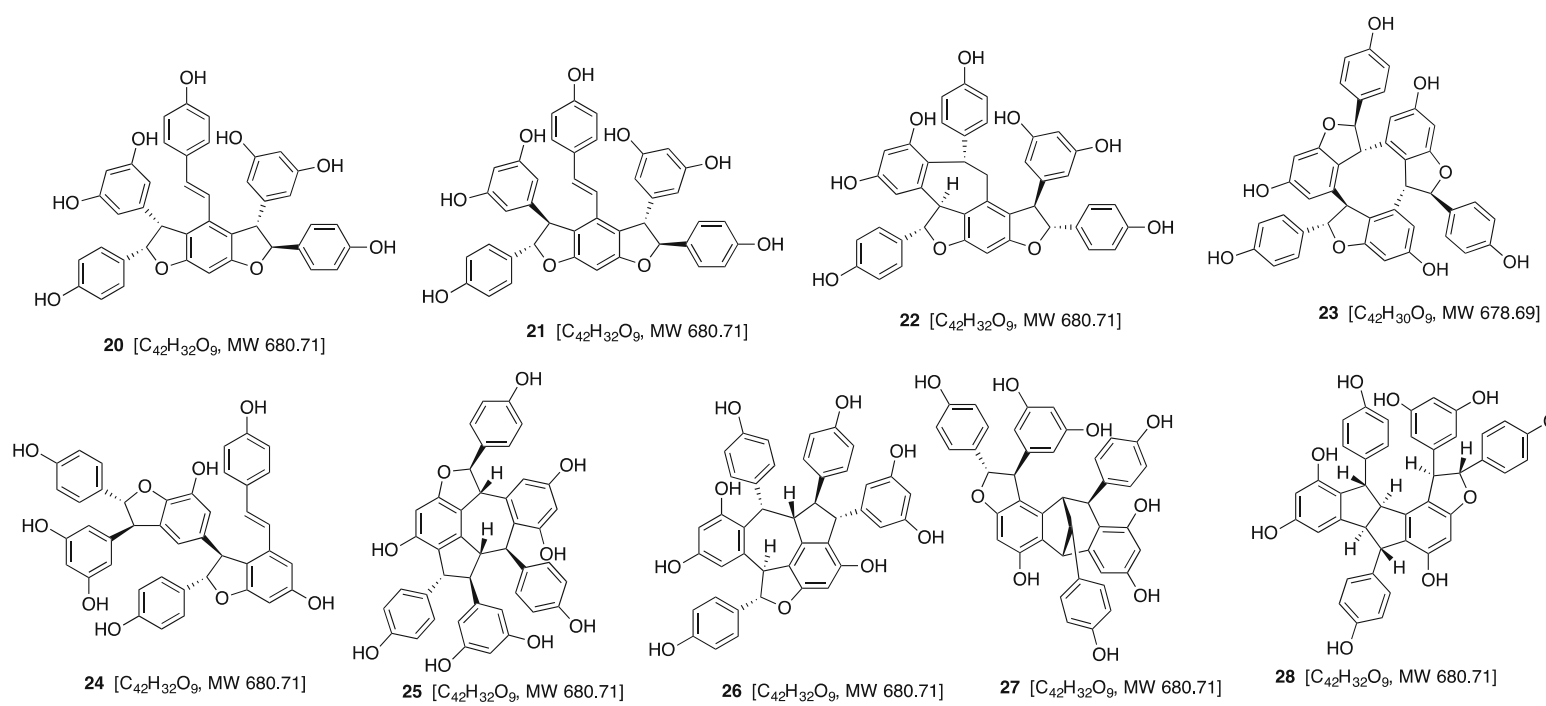

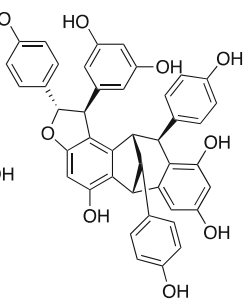

$27\left[\mathrm{C}_{42} \mathrm{H}_{32} \mathrm{O}_{9}, \mathrm{MW} 680.71\right]$

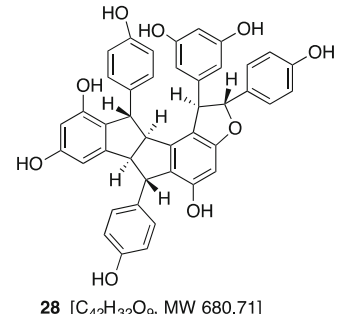

$28\left[\mathrm{C}_{42} \mathrm{H}_{32} \mathrm{O}_{9}, \mathrm{MW} 680.71\right]$

Fig. 5 Molecular structures of stilbene trimers isolated form edible berries: 20: amurensin B, 21: gnetin H, 22: vitisin E, 23: $\alpha$ viniferin, 24: miyabenol C, 25: dividol A, 26: amurensin G, 27: ampelopsin G (wilsonol B), 28: wilsonol A

(He et al. 2009a, b; Sun et al. 2006; Jiang et al. 2012), silica gel (Jiang et al. 2012) or C18 solid-phase extraction (Kiselev et al. 2017).

Many analytical methods with various detection techniques are reported for the separation and identification of individual stilbenes in edible berries, such as liquid chromatography-tandem mass spectrometry (LC-MS/MS) (Vrhovsek et al. 2012), liquid chromatography-mass spectrometry (LC-MS) (Može et al. 2011), liquid chromatography with dual detection by a photodiode array and quadrupole time-of-flight mass spectrometry (LC-PDA-QTOF/MS) (Samoticha et al. 2017), high-pressure liquid chromatography-mass spectrometry (HPLC-MS) (Bavaresco et al. 2002; Jiang et al. 2012; Kiselev et al. 2017), high-pressure liquid chromatography with diode array detection (HPLC-DAD) (Bavaresco et al. 2002; Sun et al. 2006; Vilanova et al. 2015; Guerrero et al. 2010a, 2016), high-pressure liquid chromatography with UV detection (HPLC-UV) (Vincenzi et al. 2013; Kawakami et al. 2014; He et al. 2009a), ultra-high-pressure liquid chromatography quadrupole time-of-flight mass spectrometry (UHPLC/QTOF/MS) (Flamini et al. 2016; De Rosso et al. 2016), ultra-performance liquid chromatography with dual detection by a photodiode array and fluorescence detectors (UPLC/DAD/FL) (Samoticha et al. 2017), ultra-performance liquid chromatography with dual detection by a diode array and tandem quadrupole mass spectrometry (UPLC/
DAD/TQD) (Guerrero et al. 2010a), gas chromatography-mass spectrometry (GC-MS) (Ragab et al. 2006; Viñas et al. 2009, 2011), gas chromatographymass spectrometry with selected ion monitoring (GCMS SIM) (Rimando and Cody 2005), gas-liquid chromatography with flame ionization detection (GLC-FID) (Moriartry et al. 2001), capillary electrophoresis (CE) (Ehala et al. 2005) and high-speed counter-current chromatography (HSCC) (He et al. 2009b). Various methods are used for analyses of stilbenes contents in the edible berries are different which could also contribute to the observed variability in the published results. However, the most commonly used methods for stilbenes analysis in different edible berries is normal- and reverse-phase liquid chromatography connected to a diode array detector (DAD) or mass spectrometry (MS). To improve the identification of stilbenes the ultra-performance LC (UPLC) technique coupled with QTOF-MS has been used due to higher resolution and sensitivity of analysis (Flamini et al. 2016) (Table 1, entry no. 24, 26, 27).

Gas chromatography coupled with mass spectrometry (GC-MS) has also been applied for the analyses of stilbenes (Ragab et al. 2006; Rimando and Cody 2005; Viñas et al. 2009, 2011). Prior to GC-MS, the derivatization of hydroxy groups in stilbenes was performed in order to reduce polarity and increase volatility, and simultaneously, thermal stability of 

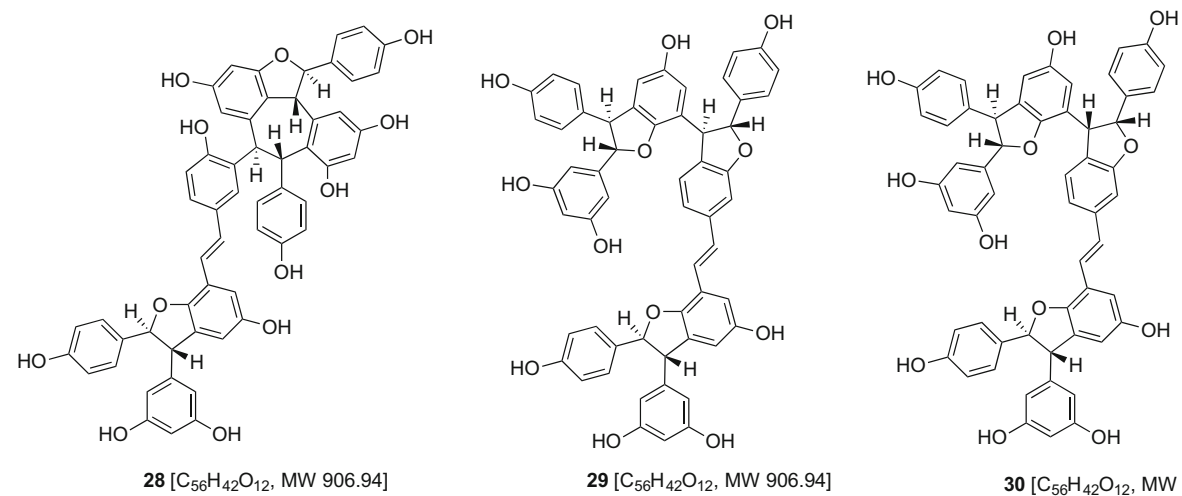

$29\left[\mathrm{C}_{56} \mathrm{H}_{42} \mathrm{O}_{12}, \mathrm{MW}\right.$ 906.94]

$30\left[\mathrm{C}_{56} \mathrm{H}_{42} \mathrm{O}_{12}, \mathrm{MW}\right.$ 906.94]
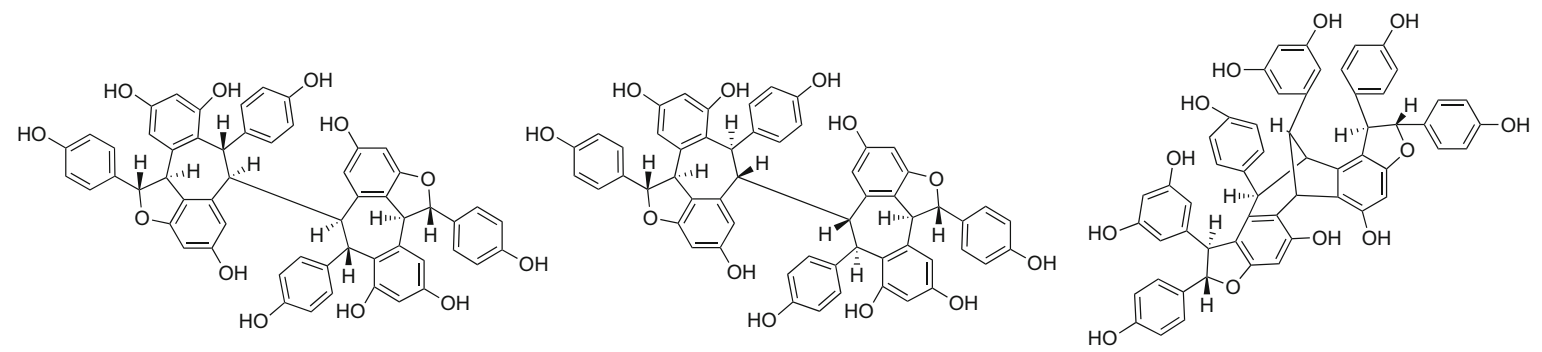

$31\left[\mathrm{C}_{56} \mathrm{H}_{42} \mathrm{O}_{12}, \mathrm{MW} 906.94\right]$

$32\left[\mathrm{C}_{56} \mathrm{H}_{42} \mathrm{O}_{12}, \mathrm{MW}\right.$ 906.94]

$33\left[\mathrm{C}_{56} \mathrm{H}_{42} \mathrm{O}_{12}, \mathrm{MW}\right.$ 906.94]

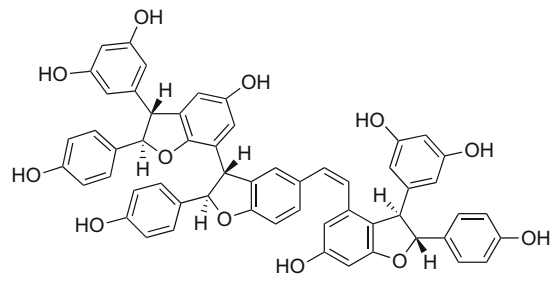

$34\left[\mathrm{C}_{56} \mathrm{H}_{42} \mathrm{O}_{12}, \mathrm{MW}\right.$ 906.94]

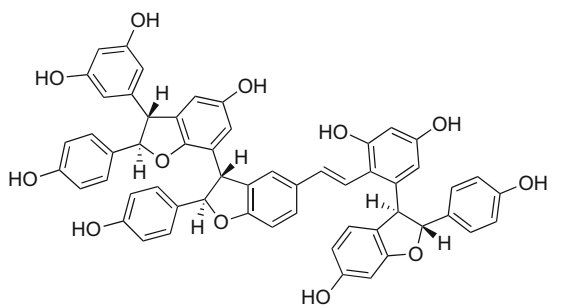

$35\left[\mathrm{C}_{56} \mathrm{H}_{42} \mathrm{O}_{12}, \mathrm{MW}\right.$ 906.94]

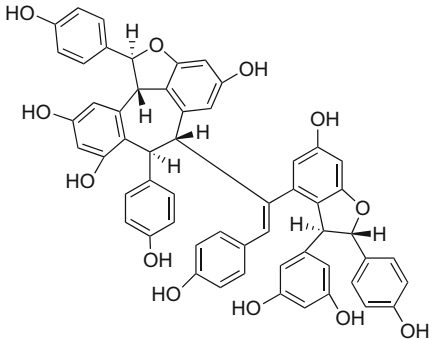

$36\left[\mathrm{C}_{56} \mathrm{H}_{42} \mathrm{O}_{12}, \mathrm{MW}\right.$ 906.94]

Fig. 6 Molecular structures of stilbene tetramers isolated form edible berries: 28: vitisin A, 29: vitisin B, 30: vitisin C, 31: hopeaphenol, 32: isohopeaphenol, 33: vaticanol C, 34: wilsonol C, 35: heyneanol A, 36: diviniferin B

metabolites. Stilbenes derivatization was based on silylation reactions by means of $\mathrm{N}, \mathrm{O}$-bis(trimethylsilyl)trifluoroacetamide (BSTFA) or $N$-methyl- $N$ (trimethylsilyl)-trifluoroacetamide (MSTFA) (Rimando and Cody 2005; Ragab et al. 2006; Viñas et al. 2009, 2011). However, due to the lower detectability of this technique only mono-stilbenes were determined such as $E$ - and $Z$-resveratrol, $E$ - and $Z$-piceid, pterostilbene and piceatannol.

Two strategies have been applied to identify stilbenes. In the case of known compounds, the identification was based on comparison of their retention times and MS or MS/MS data with those of standards. For unknown stilbenes, the characterization was performed by IR, MS, UV-Vis and NMR methods.

It is well known that the highest concentrations of stilbenes are in berries seeds and skins (Sun et al. 2006; Babazadeh et al. 2017). The amount of $E$ resveratrol in grape skin is approximately three times higher than in pulp (Babazadeh et al. 2017). Among the analysed edible berries, the highest concentration of stilbenes was found in the seeds of passion fruit (Passiflora edulis) (Kawakami et al. 2014) by applying $80 \% \mathrm{EtOH}$ as the solvent for extraction. However, the highest concentration of $E$-resveratrol in the skin has been found in table grapes (Vitis vinifera L.) (Ragab et al. 2006) by using extraction with ethyl acetate at $70{ }^{\circ} \mathrm{C}$. 


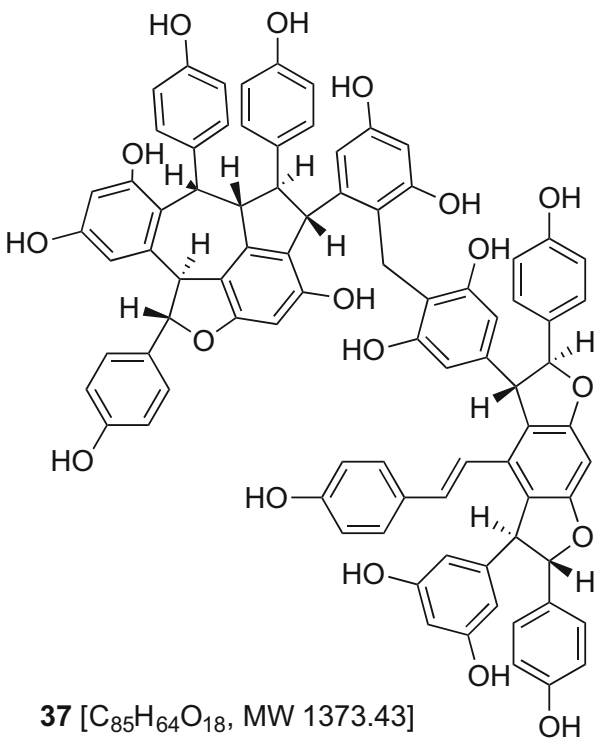

Fig. 7 Molecular structure of stilbene hexamer isolated from edible berries: 37: chunganenol

\section{Influence of external stimuli on the presence of stilbenes in grapes}

Because stilbenes are secondary metabolites in edible berries, their quantitative and qualitative composition depends on many factors, including the cultivar, genotype, type of soil, climatic conditions, developmental stage, agronomic management, storage conditions (time, temperature) and postharvest treatments. In addition, there are other external stimuli (stress factors) that activate defence mechanisms in fruits responsible for the accumulation of stilbene as phytoalexins. The defence mechanism may be induced by abiotic elicitors such as UV irradiation, ozone, ultrasonication, methyl jasmonate, chitosan and visible light or biotic elicitors such as Aspergillus carbonarius and Botrytis cinerea. Recent review has discussed the influence of external stimuli on resveratrol synthesis in grapes (Hasan and Bae 2017). Various abiotic and biotic stress conditions have significant influence on the quantitative and qualitative composition of stilbenes in grapes, which are presented in Table 2 .

\section{Abiotic preharvest treatments of grape}

Preharvest UV-C treatment of the Crimson seedless variety was applied daily for 3 days before harvest
(Table 2, no. 1) and resulted in an increased concentration of stilbenes (Guerrero et al. 2016). The maximum content of $E$-resveratrol and $E$-piceatannol was achieved $24 \mathrm{~h}$ after each daily treatment. However, the $\varepsilon$-viniferin concentration was maximal at 48 and $72 \mathrm{~h}$. In the case of $E$-piceid, Z-piceid and $\omega$ viniferin, the maximum concentration was achieved at $72 \mathrm{~h}$. The maximal contents of $E$-resveratrol, $Z$-piceid, $E$-piceid, $E$-piceatannol, $\varepsilon$-viniferin, $\omega$-viniferin, isohopeaphenol and stilbenoids were 12-, 9-, 5-, 4-, 7-, 4-, 3 - and 4-fold increased over those in the control sample. After daily periodic preharvest treatment of berries, the $E$-resveratrol content increased 83 -fold in comparison with 18 -fold growth for a single UV-C irradiation over the initial concentration. The first and the second treatments significantly increased the stilbene content, but the third daily treatment might have been important for maintaining their concentration. First, the biosynthesis of $E$-resveratrol is induced, and then the compound is glycosylated to E-piceid, which, under UV-C irradiation from the daily preharvest treatment, is transformed into $Z$-piceid.

The maximum $E$-resveratrol concentration detected in grape after UV-C treatment depends on its initial concentration, which correlates with the developmental stage of berries (Guerrero et al. 2010a, b). In another work (Table 2, no. 2), UV-C light preharvest treatment was applied on different days before grape ripeness to establish the optimum application day to reach the maximum $E$-resveratrol concentration. Due to UV-C irradiation, the highest $E$-resveratrol concentration in Red Globe grapes was achieved 3 days before harvest and was 46-fold higher than that observed in the non-treated control sample. However, at harvest, the $E$-resveratrol content was only 8.8 times higher than that in the control sample. UV-C treatment of berries 1 day before harvest resulted in 26 times higher $E$-resveratrol concentrations (Table 2, no. 2). The maximum $E$-resveratrol and $\varepsilon$-viniferin content was achieved when the UV-C dose was approximately $10,000 \mathrm{~J} / \mathrm{m}^{2}$. Both the dose and the application method, in terms of output power and exposure time, are key factors determining the final stilbene content. Treatment with an output power and exposure time of $1040 \mathrm{~W}$ and $5 \mathrm{~min}$, respectively, was selected as the most suitable condition for the UV-C treatment.

According to the literature, UV-C irradiation of edible berries effectively induces stilbene biosynthesis (Liu et al. 2010; Wang et al. 2010; Crupi et al. 2013). 
Table 1 Preparation conditions and analytical methods for stilbene separation and identification from edible berries

\begin{tabular}{|c|c|c|c|c|c|}
\hline No. & Sample & Preparation conditions & $\begin{array}{l}\text { Analytical } \\
\text { method }\end{array}$ & Stilbenes: concentration $(\mu \mathrm{g} / \mathrm{g})$ & References \\
\hline 1 & $\begin{array}{l}\text { Bilberry } \\
\text { (Vaccinium } \\
\quad \text { myrtillus L.) }\end{array}$ & $\begin{array}{l}\text { 1. Material: frozen fruits } \\
\text { 2. Solvent: } \mathrm{MeOH} \\
\text { 3. Conditions: room temperature }\end{array}$ & LC-MS & $E$-resveratrol: $2 \mu \mathrm{g} / \mathrm{g}$ fw & $\begin{array}{l}\text { Može et al. } \\
\text { (2011) }\end{array}$ \\
\hline 2 & $\begin{array}{l}\text { Bilberry } \\
\text { (Vaccinium } \\
\quad \text { myrtillus L.) } \\
\text { Cultivar: } \\
\text { wild }\end{array}$ & $\begin{array}{l}\text { Method used for berries from US } \\
\text { location } \\
\text { 1. Material: lyophilized berries } \\
\text { 2. Solvent: } \mathrm{MeOH} / \text { acetone/ } \mathrm{H}_{2} \mathrm{O} / \\
\mathrm{CH}_{3} \mathrm{COOH}(40: 40: 20: 0.1) \\
\text { 3. Conditions: } 40{ }^{\circ} \mathrm{C}, 1000 \mathrm{psi} \\
\text { 4. Purification: extraction with EtOAc } \\
\text { 5. Derivatization: MSTFA/DFA/MeOH } \\
\text { (3.5:1:0.5) } \\
\text { Method used for berries from Canada } \\
\text { 1. Material: frozen berries } \\
\text { 2. Solvent: MeOH/acetone/ }{ }_{2} \mathrm{O} / \\
\text { HCOOH (40:40:20:0.1) } \\
\text { 1. Conditions: room temperature } \\
\text { 2. Purification: C18 solid-phase } \\
\text { extraction } \\
\text { 3. Derivatization: MSTFA/DFA/MeOH } \\
\text { (3.5:1:0.5) }\end{array}$ & $\begin{array}{l}\text { GC-MS } \\
\text { SIM }\end{array}$ & $\begin{array}{l}\text { Resveratrol: } \\
0.768 \mu \mathrm{g} / \mathrm{g} \mathrm{dw}\end{array}$ & $\begin{array}{l}\text { Rimando and } \\
\text { Cody (2005) }\end{array}$ \\
\hline 3 & $\begin{array}{l}\text { Bilberry } \\
\text { (Vaccinium } \\
\quad \text { myrtillus L.) }\end{array}$ & $\begin{array}{l}\text { Method I: The microwave-assisted } \\
\text { extraction } \\
\text { 1. Material: frozen berries } \\
\text { 2. Solvent: EtOH/ } \mathrm{H}_{2} \mathrm{O}(7: 3) \\
\text { 3. Conditions: } 180 \mathrm{~W} \\
\text { 4. Purification: } \mathrm{C} 18 \text { solid-phase } \\
\text { extraction } \\
\text { Method II: The ultrasonic extraction } \\
\text { 1. Material: frozen berries } \\
\text { 2. Solvent: } \mathrm{MeOH} / \mathrm{H}_{2} \mathrm{O}(1: 1), \mathrm{MeOH} / \\
\mathrm{H}_{2} \mathrm{O} \text { (7:3), and EtOH} / \mathrm{H}_{2} \mathrm{O}(7: 3) \\
\text { 3. Conditions: room temperature in } \\
\text { ultrasonic bath } \\
\text { 4. Purification: } \mathrm{C} 18 \text { solid-phase } \\
\text { extraction }\end{array}$ & CE & $\begin{array}{l}E \text {-resveratrol: } \\
\quad 6.78 \mu \mathrm{g} / \mathrm{g} \mathrm{fw}\end{array}$ & $\begin{array}{l}\text { Ehala et al. } \\
\text { (2005) }\end{array}$ \\
\hline 4 & $\begin{array}{l}\text { Blueberries } \\
\text { (Vaccinium } \\
\text { corymbosum L.) }\end{array}$ & $\begin{array}{l}\text { 1. Material: frozen fruits } \\
\text { 2. Solvent: } \mathrm{MeOH} \\
\text { 3. Conditions: room temperature }\end{array}$ & LC-MS & $E$-resveratrol: $4 \mu \mathrm{g} / \mathrm{g}$ fw & $\begin{array}{l}\text { Može et al. } \\
\text { (2011) }\end{array}$ \\
\hline
\end{tabular}


Table 1 continued

\begin{tabular}{|c|c|c|c|c|c|}
\hline No. & Sample & Preparation conditions & $\begin{array}{l}\text { Analytical } \\
\text { method }\end{array}$ & Stilbenes: concentration $(\mu \mathrm{g} / \mathrm{g})$ & References \\
\hline 5 & $\begin{array}{l}\text { Highbush blueberry } \\
\text { (Vaccinium corymbosum L.) } \\
\text { Cultivars: } \\
\text { Bluecrop } \\
\text { wild }\end{array}$ & $\begin{array}{l}\text { Method used for berries from } \\
\text { US location } \\
\text { 1. Material: lyophilized berries } \\
\text { 2. Solvent: } \mathrm{MeOH} / \text { acetone/ } \mathrm{H}_{2} \mathrm{O} / \\
\mathrm{CH}_{3} \mathrm{COOH}(40: 40: 20: 0.1) \\
\text { 3. Conditions: } 40{ }^{\circ} \mathrm{C}, 1000 \mathrm{psi} \\
\text { 4. Purification: extraction with } \\
\text { EtOAc } \\
\text { 5. Derivatization: MSTFA/DFA/ } \\
\text { MeOH (3.5:1:0.5) } \\
\text { Method used for berries from } \\
\text { Canada } \\
\text { 1. Material: frozen berries } \\
\text { 2. Solvent: MeOH/acetone/ } \mathrm{H}_{2} \mathrm{O} / \\
\text { HCOOH ( } 40: 40: 20: 0.1) \\
\text { 3. Conditions: room temperature } \\
\text { 4. Purification: C18 solid-phase } \\
\text { extraction } \\
\text { 5. Derivatization: MSTFA/DFA/ } \\
\text { MeOH (3.5:1:0.5) }\end{array}$ & $\begin{array}{c}\text { GC-MS } \\
\text { SIM }\end{array}$ & $\begin{array}{l}\text { Bluecrop from } \\
\text { conventional } \\
\text { farming } \\
\text { Resveratrol: } \\
0.853 \mu \mathrm{g} / \mathrm{g} \mathrm{dw} \\
\text { Piceatannol: } \\
0.422 \mu \mathrm{g} / \mathrm{g} \mathrm{dw} \\
\text { Bluecrop from sustainable } \\
\text { farming } \\
\text { Resveratrol: } \\
0.327 \mu \mathrm{g} / \mathrm{g} \mathrm{dw} \\
\text { Piceatannol: } \\
0.186 \mu \mathrm{g} / \mathrm{g} \mathrm{dw} \\
\text { Wild } \\
\text { Resveratrol: } \\
1.074 \mu \mathrm{g} / \mathrm{g} \mathrm{dw}\end{array}$ & $\begin{array}{l}\text { Rimando } \\
\text { and Cody } \\
(2005)\end{array}$ \\
\hline 6 & $\begin{array}{l}\text { Rabbiteye blueberry } \\
\text { (Vaccinium ashei Reade) } \\
\text { Cultivars: } \\
\text { Tifblue } \\
\text { Climax } \\
\text { Premier }\end{array}$ & $\begin{array}{l}\text { Method used for berries from } \\
\text { US location } \\
\text { 1. Material: lyophilized berries } \\
\text { 2. Solvent: } \mathrm{MeOH} / \text { acetone/ } \mathrm{H}_{2} \mathrm{O} / \\
\mathrm{CH}_{3} \mathrm{COOH}(40: 40: 20: 0.1) \\
\text { 3. Conditions: } 40{ }^{\circ} \mathrm{C}, 1000 \mathrm{psi} \\
\text { 4. Purification: extraction with } \\
\text { EtOAc } \\
\text { 5. Derivatization: MSTFA/DFA/ } \\
\text { MeOH (3.5:1:0.5) } \\
\text { Method used for berries from } \\
\text { Canada } \\
\text { 1. Material: frozen berries } \\
\text { 2. Solvent: MeOH/acetone/ } \mathrm{H}_{2} \mathrm{O} / \\
\text { HCOOH (40:40:20:0.1) } \\
\text { 3. Conditions: room temperature } \\
\text { 4. Purification: C18 solid-phase } \\
\text { extraction } \\
\text { 5. Derivatization: MSTFA/DFA/ } \\
\text { MeOH (3.5:1:0.5) }\end{array}$ & $\begin{array}{c}\text { GC-MS } \\
\text { SIM }\end{array}$ & $\begin{array}{l}\text { Tifblue } \\
\text { Resveratrol: } \\
0.106 \mu \mathrm{g} / \mathrm{g} \mathrm{dw} \\
\text { Pterostilbene: } \\
0.151 \mu \mathrm{g} / \mathrm{g} \mathrm{dw} \\
\text { Climax } \\
\text { Resveratrol: } \\
0.390 \mu \mathrm{g} / \mathrm{g} \mathrm{dw} \\
\text { Pterostilbene: } \\
0.099 \mu \mathrm{g} / \mathrm{g} \mathrm{dw} \\
\text { Premier } \\
\text { Resveratrol: } \\
0.007 \mu \mathrm{g} / \mathrm{g} \mathrm{dw}\end{array}$ & $\begin{array}{l}\text { Rimando } \\
\text { and Cody } \\
(2005)\end{array}$ \\
\hline
\end{tabular}


Table 1 continued

\begin{tabular}{|c|c|c|c|c|c|}
\hline No. & Sample & Preparation conditions & $\begin{array}{l}\text { Analytical } \\
\text { method }\end{array}$ & Stilbenes: concentration $(\mu \mathrm{g} / \mathrm{g})$ & References \\
\hline 7 & $\begin{array}{l}\text { Cowberry } \\
\text { (Vaccinium Vitis- } \\
\quad \text { idaea L.) }\end{array}$ & $\begin{array}{l}\text { Method I: The microwave-assisted } \\
\text { extraction } \\
\text { 1. Material: frozen berries } \\
\text { 2. Solvent: EtOH/ } \mathrm{H}_{2} \mathrm{O}(7: 3) \\
\text { 3. Conditions: } 180 \mathrm{~W} \\
\text { 4. Purification: } \mathrm{C} 18 \text { solid-phase extraction } \\
\text { Method II: The ultrasonic extraction } \\
\text { 1. Material: frozen berries } \\
\text { 2. Solvent: } \mathrm{MeOH} / \mathrm{H}_{2} \mathrm{O}(1: 1), \mathrm{MeOH} / \mathrm{H}_{2} \mathrm{O} \\
\text { (7:3), and } \mathrm{EtOH} / \mathrm{H}_{2} \mathrm{O}(7: 3) \\
\text { 3. Conditions: room temperature in } \\
\text { ultrasonic bath } \\
\text { 4. Purification: } \mathrm{C} 18 \text { solid-phase extraction }\end{array}$ & $\mathrm{CE}$ & $E$-resveratrol: $30 \mu \mathrm{g} / \mathrm{g} \mathrm{fw}$ & $\begin{array}{l}\text { Ehala } \\
\text { et al. } \\
(2005)\end{array}$ \\
\hline 8 & $\begin{array}{l}\text { Cranberry } \\
\text { (Vaccinium } \\
\text { oxycoccos) }\end{array}$ & $\begin{array}{l}\text { Method I: the microwave-assisted } \\
\text { extraction } \\
\text { 1. Material: frozen berries } \\
\text { 2. Solvent: } \mathrm{EtOH} / \mathrm{H}_{2} \mathrm{O}(7: 3) \\
\text { 3. Conditions: } 180 \mathrm{~W} \\
\text { 4. Purification: } \mathrm{C} 18 \text { solid-phase extraction } \\
\text { Method II: the ultrasonic extraction } \\
\text { 1. Material: frozen berries } \\
\text { 2. Solvent: } \mathrm{MeOH} / \mathrm{H}_{2} \mathrm{O}(1: 1), \mathrm{MeOH} / \mathrm{H}_{2} \mathrm{O} \\
\text { (7:3), and } \mathrm{EtOH} / \mathrm{H}_{2} \mathrm{O}(7: 3) \\
\text { 3. Conditions: room temperature in } \\
\text { ultrasonic bath } \\
\text { 4. Purification: } \mathrm{C} 18 \text { solid-phase extraction }\end{array}$ & $\mathrm{CE}$ & $E$-resveratrol: $19.29 \mu \mathrm{g} / \mathrm{g}$ fw & $\begin{array}{l}\text { Ehala } \\
\text { et al. } \\
(2005)\end{array}$ \\
\hline 9 & $\begin{array}{l}\text { Red currant } \\
\text { (Ribes rubrum L.) } \\
\text { Black currant } \\
\text { (Ribes nigrum L.) }\end{array}$ & $\begin{array}{l}\text { Method I: the microwave-assisted } \\
\text { extraction } \\
\text { 1. Material: frozen berries } \\
\text { 2. Solvent: } \mathrm{EtOH} / \mathrm{H}_{2} \mathrm{O}(7: 3) \\
\text { 3. Conditions: } 180 \mathrm{~W} \\
\text { 4. Purification: } \mathrm{C} 18 \text { solid-phase extraction } \\
\text { Method II: The ultrasonic extraction } \\
\text { 1. Material: frozen berries } \\
\text { 2. Solvent: } \mathrm{MeOH} / \mathrm{H}_{2} \mathrm{O}(1: 1), \mathrm{MeOH} / \mathrm{H}_{2} \mathrm{O} \\
\text { (7:3), and EtOH} / \mathrm{H}_{2} \mathrm{O}(7: 3) \\
\text { 3. Conditions: room temperature in } \\
\text { ultrasonic bath } \\
\text { 4. Purification: } \mathrm{C} 18 \text { solid-phase extraction }\end{array}$ & $\mathrm{CE}$ & $\begin{array}{l}\text { Red currant } \\
\text { E-resveratrol: } \\
15.72 \mu \mathrm{g} / \mathrm{g} \text { fw } \\
\text { Black currant } \\
\text { E-resveratrol: nd }\end{array}$ & $\begin{array}{l}\text { Ehala } \\
\text { et al. } \\
(2005)\end{array}$ \\
\hline
\end{tabular}


Table 1 continued

\begin{tabular}{|c|c|c|c|c|c|}
\hline No. & Sample & Preparation conditions & $\begin{array}{l}\text { Analytical } \\
\text { method }\end{array}$ & Stilbenes: concentration $(\mu \mathrm{g} / \mathrm{g})$ & References \\
\hline 10 & $\begin{array}{l}\text { Deerberry } \\
\text { (Vaccinium } \\
\quad \text { stamineum L.) } \\
\text { Cultivars: } \\
\text { wild } \\
\text { SHF3A-2-108 } \\
\text { B-76 }\end{array}$ & $\begin{array}{l}\text { Method used for berries from US location } \\
\text { 1. Material: lyophilized berries } \\
\text { 2. Solvent: } \mathrm{MeOH} / \text { acetone/ } \mathrm{H}_{2} \mathrm{O} / \\
\mathrm{CH}_{3} \mathrm{COOH}(40: 40: 20: 0.1) \\
\text { 3. Conditions: } 40{ }^{\circ} \mathrm{C}, 1000 \mathrm{psi} \\
\text { 4. Purification: extraction with EtOAc } \\
\text { 5. Derivatization: MSTFA/DFA/MeOH } \\
\text { (3.5:1:0.5) } \\
\text { Method used for berries from Canada } \\
\text { 1. Material: frozen berries } \\
\text { 2. Solvent: MeOH/acetone/ } \mathrm{H}_{2} \mathrm{O} / \mathrm{HCOOH} \\
\text { (40:40:20:0.1) } \\
\text { 3. Conditions: room temperature } \\
\text { 4. Purification: } \mathrm{C} 18 \text { solid-phase extraction } \\
\text { 5. Derivatization: MSTFA/DFA/MeOH } \\
\text { (3.5:1:0.5) }\end{array}$ & $\begin{array}{c}\text { GC-MS } \\
\text { SIM }\end{array}$ & $\begin{array}{l}\text { Wild } \\
\text { Resveratrol: } 0.204 \mu \mathrm{g} / \mathrm{g} \mathrm{dw} \\
\text { SHF3A-2-108 } \\
\text { Resveratrol: } 0.115 \mu \mathrm{g} / \mathrm{g} \mathrm{dw} \\
\text { Pterostilbene: } 0.520 \mu \mathrm{g} / \mathrm{g} \mathrm{dw} \\
\text { B-76 } \\
\text { Resveratrol: } 0.503 \mu \mathrm{g} / \mathrm{g} \mathrm{dw} \\
\text { Piceatannol: } 0.195 \mu \mathrm{g} / \mathrm{g} \mathrm{dw}\end{array}$ & $\begin{array}{l}\text { Rimando } \\
\text { and } \\
\text { Cody } \\
(2005)\end{array}$ \\
\hline 11 & $\begin{array}{l}\text { Table grapes } \\
\text { (Vitis vinifera L.) } \\
\text { Californian } \\
\text { cultivars: } \\
\text { Black Corinth } \\
\text { Flame Seedless } \\
\text { skin }\end{array}$ & $\begin{array}{l}\text { 1. Material: fresh skin } \\
\text { 2. Solvent: } \mathrm{MeOH} / 0.1 \% \mathrm{HCl} \\
\text { 3. Conditions: room temperature } \\
\text { 4. Purification: extraction with EtOAc }\end{array}$ & GLC-FID & $\begin{array}{l}\text { Resveratrol } \\
\text { Black Corinth cultivar } \\
\text { Non-irradiated fruits: } \\
0-25.1 \mu \mathrm{g} / \mathrm{g} \text { fw } \\
\text { Irradiated fruits: } 0.9-33.2 \mu \mathrm{g} / \mathrm{g} \mathrm{fw} \\
\text { Flame Seedless cultivar } \\
\text { Non-irradiated fruits: } \\
\text { 1-13.2 } \mu \mathrm{g} / \mathrm{g} \text { fw } \\
\text { Irradiated fruits: } 1.8-57.3 \mu \mathrm{g} / \mathrm{g} \mathrm{fw}\end{array}$ & $\begin{array}{l}\text { Moriartry } \\
\text { et al. } \\
\text { (2001) }\end{array}$ \\
\hline 12 & $\begin{array}{l}\text { Grapes } \\
\text { (Vitis vinifera } \mathrm{L} . \text {.) } \\
\text { Cultivar: } \\
\text { Cabernet } \\
\text { sauvignon }\end{array}$ & $\begin{array}{l}\text { 1. Material: fresh berries (without seeds) } \\
\text { 2. Solvent: } 95 \% \mathrm{MeOH} \\
\text { 3. Conditions: room temperature } \\
\text { 4. Purification: EtOAc/5\% } \mathrm{NaHCO}_{3}(1: 1)\end{array}$ & $\begin{array}{l}\text { HPLC- } \\
\text { DAD } \\
\text { HPLC-MS }\end{array}$ & $\begin{array}{l}E \text {-resveratrol: } 0.297 \mu \mathrm{g} / \mathrm{g} \mathrm{fw} \\
E \text {-piceid: } 0.097 \mu \mathrm{g} / \mathrm{g} \mathrm{fw} \\
\text { Piceatannol: } 0.052 \mu \mathrm{g} / \mathrm{g} \mathrm{fw} \\
Z \text {-resveratrol: nd } \\
Z \text {-piceid: nd }\end{array}$ & $\begin{array}{l}\text { Bavaresco } \\
\text { et al. } \\
\text { (2002) }\end{array}$ \\
\hline 13 & $\begin{array}{l}\text { Grapes } \\
\text { (Vitis vinifera } \text { L.) } \\
\text { Cultivars: } \\
\text { Cabernet } \\
\text { Pinot Noir } \\
\text { Merlot } \\
\text { Table grapes }\end{array}$ & $\begin{array}{l}\text { Method used for berries from US location } \\
\text { 1. Material: lyophilized berries } \\
\text { 2. Solvent: } \mathrm{MeOH} / \text { acetone/ } \mathrm{H}_{2} \mathrm{O} / \\
\mathrm{CH}_{3} \mathrm{COOH}(40: 40: 20: 0.1) \\
\text { 3. Conditions: } 40{ }^{\circ} \mathrm{C}, 1000 \mathrm{psi} \\
\text { 4. Purification: extraction with EtOAc } \\
\text { 5. Derivatization: MSTFA/DFA/MeOH } \\
\text { (3.5:1:0.5) } \\
\text { Method used for berries from Canada } \\
\text { 1. Material: frozen berries } \\
\text { 2. Solvent: MeOH/acetone/ } \mathrm{H}_{2} \mathrm{O} / \mathrm{HCOOH} \\
\text { (40:40:20:0.1) } \\
\text { 3. Conditions: room temperature } \\
\text { 4. Purification: } \mathrm{C} 18 \text { solid-phase extraction } \\
\text { 5. Derivatization: MSTFA/DFA/MeOH } \\
\text { (3.5:1:0.5) }\end{array}$ & $\begin{array}{c}\text { GC-MS } \\
\text { SIM }\end{array}$ & $\begin{array}{l}\text { Cabernet } \\
\text { Resveratrol: } 2.475 \mu \mathrm{g} / \mathrm{g} \mathrm{dw} \\
\text { Pinot Noir } \\
\text { Resveratrol: } 5.746 \mu \mathrm{g} / \mathrm{g} \mathrm{dw} \\
\text { Merlot } \\
\text { Resveratrol: } 6.356 \mu \mathrm{g} / \mathrm{g} \mathrm{dw} \\
\text { Table grapes } \\
\text { Resveratrol: } 6.471 \mu \mathrm{g} / \mathrm{g} \mathrm{dw}\end{array}$ & $\begin{array}{l}\text { Rimando } \\
\text { and } \\
\text { Cody } \\
(2005)\end{array}$ \\
\hline
\end{tabular}


Table 1 continued

\begin{tabular}{|c|c|c|c|c|c|}
\hline No. & Sample & Preparation conditions & $\begin{array}{l}\text { Analytical } \\
\text { method }\end{array}$ & Stilbenes: concentration $(\mu \mathrm{g} / \mathrm{g})$ & References \\
\hline 14 & $\begin{array}{l}\text { Table grapes } \\
\text { (Vitis } \\
\quad \text { vinifera } \\
\text { L.) } \\
\text { seedless red }\end{array}$ & $\begin{array}{l}\text { 1. Material: lyophilized tomato or grape skin } \\
\text { 2. Solvent: EtOAc } \\
\text { 3. Conditions: } 70{ }^{\circ} \mathrm{C} \\
\text { 4. Derivatization: MSTFA/pyridine }\end{array}$ & GS-MS & $\begin{array}{l}Z \text {-resveratrol: } 20 \mu \mathrm{g} / \mathrm{g} \mathrm{dw} \\
E \text {-resveratrol: } 2680 \mu \mathrm{g} / \mathrm{g} \mathrm{dw} \\
Z \text {-piceid: } 30 \mu \mathrm{g} / \mathrm{g} \mathrm{dw} \\
E \text {-piceid: } 50 \mu \mathrm{g} / \mathrm{g} \mathrm{dw}\end{array}$ & $\begin{array}{l}\text { Ragab } \\
\text { et al. } \\
(2006)\end{array}$ \\
\hline 15 & $\begin{array}{l}\text { Grapes } \\
\text { (Vitis } \\
\quad \text { vinifera } \\
\quad \text { L.) } \\
\text { Cultivars: } \\
\text { Castelao } \\
\text { Syrah } \\
\text { Tinta Roriz } \\
\text { seeds } \\
\text { skin }\end{array}$ & 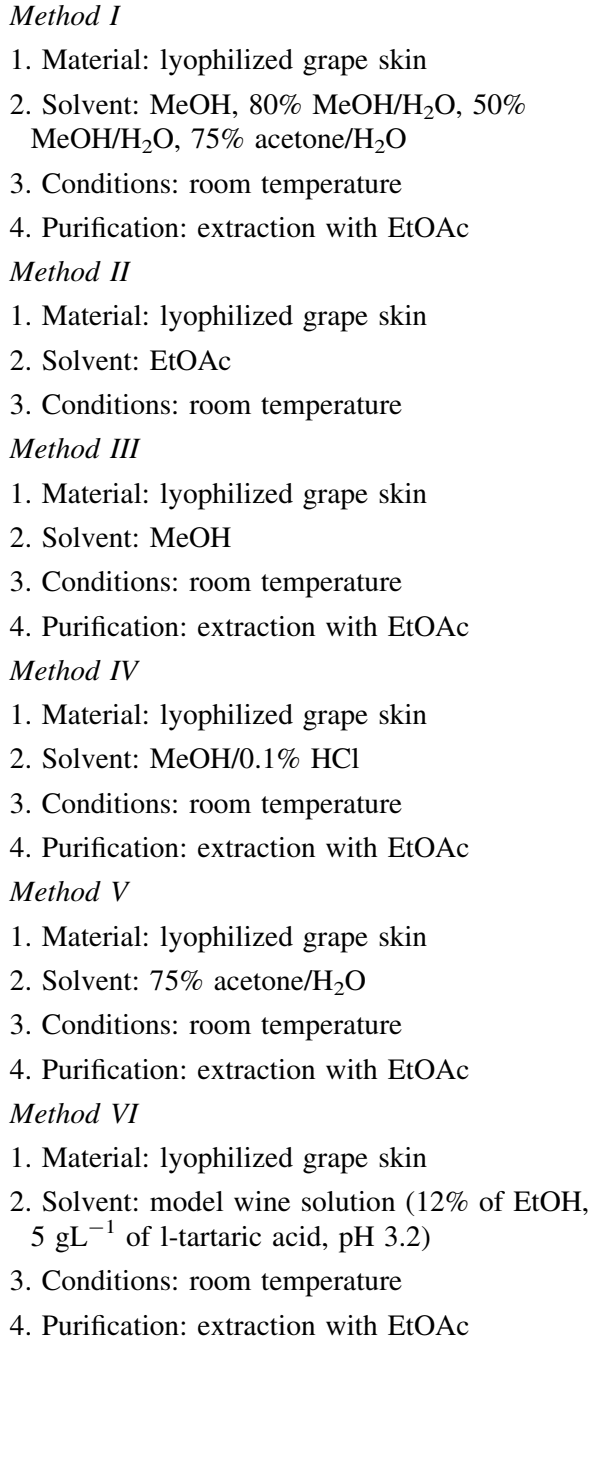 & $\begin{array}{c}\text { HPLC- } \\
\text { DAD }\end{array}$ & $\begin{array}{l}\text { Method I } \\
\text { E-resveratrol: } \sim 40 \mu \mathrm{g} / \mathrm{g} \mathrm{fw} \\
\text { E-piceid: } \sim 70 \mu \mathrm{g} / \mathrm{g} \mathrm{fw} \\
\text { Z-piceid: } \sim 130 \mu \mathrm{g} / \mathrm{g} \mathrm{fw} \\
\text { Method II } \\
\text { E-resveratrol: } \sim 40 \mu \mathrm{g} / \mathrm{g} \mathrm{fw} \\
\text { E-piceid: } \sim 10 \mu \mathrm{g} / \mathrm{g} \mathrm{fw} \\
\text { Z-piceid: } \sim 20 \mu \mathrm{g} / \mathrm{g} \mathrm{fw} \\
\text { Method III } \\
\text { E-resveratrol: } \sim 80 \mu \mathrm{g} / \mathrm{g} \mathrm{fw} \\
\text { E-piceid: } \sim 120 \mu \mathrm{g} / \mathrm{g} \mathrm{fw} \\
Z \text {-piceid: } \sim 170 \mu \mathrm{g} / \mathrm{g} \mathrm{fw} \\
\text { Method IV } \\
\text { E-resveratrol: } \sim 100 \mu \mathrm{g} / \mathrm{g} \mathrm{fw} \\
\text { E-piceid: } \sim 140 \mu \mathrm{g} / \mathrm{g} \mathrm{fw} \\
Z \text {-piceid: } \sim 200 \mu \mathrm{g} / \mathrm{g} \mathrm{fw} \\
\text { Method } V \\
\text { E-resveratrol: } \sim 30 \mu \mathrm{g} / \mathrm{g} \mathrm{fw} \\
\text { E-piceid: } \sim 20 \mu \mathrm{g} / \mathrm{g} \mathrm{fw} \\
Z \text {-piceid: } \sim 130 \mu \mathrm{g} / \mathrm{g} \mathrm{fw} \\
\text { Method VI } \\
\text { E-resveratrol: } \sim 10 \mu \mathrm{g} / \mathrm{g} \mathrm{fw} \\
\text { E-piceid: } \sim 10 \mu \mathrm{g} / \mathrm{g} \mathrm{fw} \\
Z \text {-piceid: } \sim 30 \mu \mathrm{g} / \mathrm{g} \mathrm{fw} \\
\text { Method IV } \\
\text { Seed } \\
\text { Total resveratrol: } \\
\text { 4.76 } \pm 0.25 \mu \mathrm{g} / \mathrm{g} \mathrm{dw} \\
\text { Total piceid: nd } \\
\text { Skin } \\
\text { Total resveratrol: } \\
\text { To8.50 } \pm 51.97 \mu \mathrm{g} / \mathrm{g} \mathrm{dw} \\
\text { 762.47 } \pm 164.16 \mu \mathrm{g} / \mathrm{g} \mathrm{dw}\end{array}$ & $\begin{array}{l}\text { Sun et al. } \\
\text { (2006) }\end{array}$ \\
\hline
\end{tabular}


Table 1 continued

\begin{tabular}{|c|c|c|c|c|c|}
\hline No. & Sample & Preparation conditions & $\begin{array}{l}\text { Analytical } \\
\text { method }\end{array}$ & Stilbenes: concentration $(\mu \mathrm{g} / \mathrm{g})$ & References \\
\hline 16 & $\begin{array}{l}\text { Southeast China grape } \\
\text { (Vitis chunganensis) }\end{array}$ & $\begin{array}{l}\text { 1. Material: fresh grape } \\
\text { 2. Solvent: } \mathrm{MeOH} \\
\text { 3. Conditions: room } \\
\text { temperature } \\
\text { 4. Purification: EtOAc and } \\
\text { silica gel/EtOAc/light } \\
\text { petroleum }\end{array}$ & HPLC-UV & $\begin{array}{l}\text { Chunganenol: } 8.57 \mu \mathrm{g} / \mathrm{g} \mathrm{fw} \\
\text { Amurensin B: } 242.86 \mu \mathrm{g} / \mathrm{g} \text { fw } \\
\text { Gnetin H: } 131.43 \mu \mathrm{g} / \mathrm{g} \mathrm{fw} \\
\text { E-viniferin: } 154.29 \mu \mathrm{g} / \mathrm{g} \mathrm{fw} \\
\text { Amurensin G: } 262.86 \mu \mathrm{g} / \mathrm{g} \mathrm{fw} \\
\text { Vitisin A: } 191.43 \mu \mathrm{g} / \mathrm{g} \mathrm{fw} \\
\text { Hopeaphenol: } 88.57 \mu \mathrm{g} / \mathrm{g} \mathrm{fw} \\
\text { Resveratrol: } 48.57 \mu \mathrm{g} / \mathrm{g} \mathrm{fw}\end{array}$ & $\begin{array}{l}\text { He et al. } \\
\text { (2009a) }\end{array}$ \\
\hline 17 & $\begin{array}{l}\text { Southeast China grape } \\
\text { (Vitis chunganensis) }\end{array}$ & $\begin{array}{l}\text { 1. Material: dried fruits } \\
\text { 2. Solvent: } \mathrm{MeOH} \\
\text { 3. Conditions: room } \\
\text { temperature } \\
\text { 4. Purification: extraction with } \\
\text { EtOAc }\end{array}$ & HSCCC & $\begin{array}{l}\text { Hopeaphenol: } 84.4 \mu \mathrm{g} / \mathrm{g} \mathrm{dw} \\
\text { Amurensin G: } 148.8 \mu \mathrm{g} / \mathrm{g} \mathrm{dw} \\
\text { Vitisin A: } 382.4 \mu \mathrm{g} / \mathrm{g} \mathrm{dw}\end{array}$ & $\begin{array}{l}\text { He et al. } \\
\quad(2009 b)\end{array}$ \\
\hline 18 & $\begin{array}{l}\text { Grapes } \\
\text { (Vitis vinifera L.) } \\
\text { red and white }\end{array}$ & $\begin{array}{l}\text { 1. Material: fresh grapes } \\
\text { 2. Solvent: EtOH } \\
\text { 3. Conditions: sonication at } \\
\text { room temperature } \\
\text { 4. Derivatization: BSTFA }\end{array}$ & GC-MS & $\begin{array}{l}\text { Red grapes } \\
\text { E-resveratrol: } 0.029 \mu \mathrm{g} / \mathrm{g} \mathrm{fw} \\
\text { Z-resveratrol: } 0.0028 \mu \mathrm{g} / \mathrm{g} \mathrm{fw} \\
\text { Piceatannol: } 0.024 \mu \mathrm{g} / \mathrm{g} \mathrm{fw} \\
\text { White grapes } \\
\text { E-resveratrol: } 0.0056 \mu \mathrm{g} / \mathrm{g} \mathrm{fw} \\
\text { Z-resveratrol: } \mathrm{nd} \\
\text { Piceatannol: } 0.0012 \mu \mathrm{g} / \mathrm{g} \mathrm{fw}\end{array}$ & $\begin{array}{l}\text { Viñas et al. } \\
\text { (2009) }\end{array}$ \\
\hline 19 & $\begin{array}{l}\text { Grapes } \\
\text { (3 Vitis vinifera } \\
\text { sylvestris, } \\
7 \text { Vitis vinifera sativa, } \\
2 \text { Hybrid Direct } \\
\text { Producers) }\end{array}$ & $\begin{array}{l}\text { 1. Material: frozen grapes } \\
\text { 2. Solvent: } \mathrm{Et}_{2} \mathrm{O} \\
\text { 3. Conditions: room } \\
\text { temperature }\end{array}$ & $\begin{array}{l}\text { UPLC-DAD- } \\
\text { TQD } \\
\text { identification } \\
\text { HPLC-DAD } \\
\text { determination }\end{array}$ & $\begin{array}{l}\text { Merlot: non- } U V, U V \text {-C treatment } \\
\quad(\mu \mathrm{g} / \mathrm{g} \text { fw }) \\
\text { E-resveratrol: } 2.82,9.75 \\
\text { Piceatannol: } 0.48,2.55 \\
\text { Viniferins: } 0.29,2.39 \\
\text { Syrah: non- } U V, U V \text {-C treatment } \\
\quad(\mu \mathrm{g} / \mathrm{g} \text { fw) } \\
\text { E-resveratrol: } 3.56,19.56 \\
\text { Piceatannol: } 0.46,0.31 \\
\text { Viniferins: nd, } 1.49 \\
\text { Tempranillo: } n o n-U V, U V-C \\
\quad \text { treatment ( } \mu \mathrm{g} / \mathrm{g} \text { fw) } \\
\text { E-resveratrol: } 0.31,3.78 \\
\text { Piceatannol: } \mathrm{nd}, 1.10 \\
\text { Viniferins: } 0.14,1.19\end{array}$ & $\begin{array}{l}\text { Guerrero } \\
\text { et al. } \\
\text { (2010a) }\end{array}$ \\
\hline 20 & $\begin{array}{l}\text { Grapes } \\
\text { (Vitis vinifera L.) } \\
\text { red and white }\end{array}$ & $\begin{array}{l}\text { 1. Material: fresh fruits } \\
\text { 2. Solvent: undecanone } \\
\text { 3. Conditions: } 30^{\circ} \mathrm{C} \\
\text { 4. Derivatization: BSTFA }\end{array}$ & $\mathrm{GC}-\mathrm{MS}$ & $\begin{array}{l}\text { Red grapes } \\
\text { E-resveratrol: } 1.639 \mu \mathrm{g} / \mathrm{g} \mathrm{fw} \\
\text { Z-resveratrol: } 0.405 \mu \mathrm{g} / \mathrm{g} \mathrm{fw} \\
\text { Piceatannol: } 0.374 \mu \mathrm{g} / \mathrm{g} \mathrm{fw} \\
\text { White grapes } \\
\text { E-resveratrol: } 0.239 \mu \mathrm{g} / \mathrm{g} \mathrm{fw} \\
\text { Z-resveratrol: } 0.082 \mu \mathrm{g} / \mathrm{g} \mathrm{fw} \\
\text { Piceatannol: } 0.043 \mu \mathrm{g} / \mathrm{g} \mathrm{fw}\end{array}$ & $\begin{array}{l}\text { Viñas et al. } \\
\text { (2011) }\end{array}$ \\
\hline
\end{tabular}


Table 1 continued

\begin{tabular}{|c|c|c|c|c|c|}
\hline No. & Sample & Preparation conditions & $\begin{array}{l}\text { Analytical } \\
\text { method }\end{array}$ & Stilbenes: concentration $(\mu \mathrm{g} / \mathrm{g})$ & References \\
\hline 21 & $\begin{array}{l}\text { Grapes } \\
\text { (Vitis } \\
\quad \text { vinifera } \\
\text { L.) }\end{array}$ & $\begin{array}{l}\text { 1. Material: fresh fruits } \\
\text { 2. Solvent: } \mathrm{H}_{2} \mathrm{O} / \mathrm{MeOH} / \mathrm{CHCl}_{3}(20: 40: 40) \\
\text { 3. Conditions: room temperature } \\
\text { 4. Purification: extraction with } \mathrm{H}_{2} \mathrm{O} / \mathrm{MeOH} \\
(1: 2)\end{array}$ & $\begin{array}{c}\text { LC-MS/ } \\
\text { MS }\end{array}$ & $\begin{array}{l}\text { Piceatannol: } 0.08 \mu \mathrm{g} / \mathrm{g} \mathrm{fw} \\
\text { E-piceid: } 0.27 \mu \mathrm{g} / \mathrm{g} \mathrm{fw} \\
\text { Z-piceid: } 0.02 \mu \mathrm{g} / \mathrm{g} \mathrm{fw} \\
\text { Astringin: } 0.69 \mu \mathrm{g} / \mathrm{g} \mathrm{fw} \\
\text { Isorhapontin: } 0.08 \mu \mathrm{g} / \mathrm{g} \mathrm{fw} \\
\text { E-E-viniferin: } 0.32 \mu \mathrm{g} / \mathrm{g} \mathrm{fw} \\
\text { Pallidol: } 0.04 \mu \mathrm{g} / \mathrm{g} \mathrm{fw} \\
\text { Isohopeaphenol: } 0.19 \mu \mathrm{g} / \mathrm{g} \mathrm{fw}\end{array}$ & $\begin{array}{l}\text { Vrhovsek } \\
\text { et al. } \\
\text { (2012) }\end{array}$ \\
\hline 22 & $\begin{array}{l}\text { Wild grape } \\
\text { (Vitis } \\
\quad \text { wilsonae) }\end{array}$ & $\begin{array}{l}\text { 1. Material: air-dried grapes } \\
\text { 2. Solvent: } \mathrm{MeOH} \\
\text { 3. Conditions: room temperature } \\
\text { 4. Purification: extraction with EtOAc and } \\
\text { silica gel/EtOAc/petroleum ether }\end{array}$ & HPLC-MS & $\begin{array}{l}\text { Wilsonol A: } 1.87 \mu \mathrm{g} / \mathrm{g} \mathrm{dw} \\
\text { Wilsonol B: } 0.72 \mu \mathrm{g} / \mathrm{g} \mathrm{dw} \\
\text { Wilsonol C: } 1.13 \mu \mathrm{g} / \mathrm{g} \mathrm{dw} \\
\text { Diviniferin B: } 3.03 \mu \mathrm{g} / \mathrm{g} \mathrm{dw} \\
\text { Pallidol: } 0.75 \mu \mathrm{g} / \mathrm{g} \mathrm{dw} \\
\text { E-viniferin: } 0.23 \mu \mathrm{g} / \mathrm{g} \mathrm{dw} \\
\text { Ampelopsin B: } 1.13 \mu \mathrm{g} / \mathrm{g} \mathrm{dw} \\
\text { Ampelopsin D: } 0.32 \mu \mathrm{g} / \mathrm{g} \mathrm{dw} \\
\text { Miyabenol C: } 3.75 \mu \mathrm{g} / \mathrm{g} \mathrm{dw} \\
\text { Dividol A: } 1.9 \mu \mathrm{g} / \mathrm{g} \mathrm{dw} \\
\text { Hopeaphenol: } 6.13 \mu \mathrm{g} / \mathrm{g} \mathrm{dw} \\
\text { Gnetin H: } 11.67 \mu \mathrm{g} / \mathrm{g} \mathrm{dw} \\
\text { Heyneanol A: } 4.3 \mu \mathrm{g} / \mathrm{g} \mathrm{dw} \\
\text { Ampelopsin G: } 1.87 \mu \mathrm{g} / \mathrm{g} \mathrm{dw} \\
\text { Amurensin G: } 2.78 \mu \mathrm{g} / \mathrm{g} \mathrm{dw} \\
\text { Visitin E: } 4.02 \mu \mathrm{g} / \mathrm{g} \mathrm{dw}\end{array}$ & $\begin{array}{c}\text { Jiang et al. } \\
\text { (2012) }\end{array}$ \\
\hline 23 & $\begin{array}{l}\text { Grapes } \\
\text { (Vitis } \\
\text { vinifera } \\
\text { L.) } \\
21 \text { red } \\
\text { cultivars, } \\
\text { skin }\end{array}$ & $\begin{array}{l}\text { 1. Material: skin of frozen berries } \\
\text { 2. Solvent: } \mathrm{MeOH} / \mathrm{HCl}(40 \mathrm{~mL} / 50 \mu \mathrm{L}) \\
\text { 3. Conditions: room temperature } \\
\text { 4. Purifiction: extraction with EtOAc and } \\
\text { then } \mathrm{MeOH} / \mathrm{HCOOH}\end{array}$ & HPLC-UV & $\begin{array}{l}E \text {-resveratrol: detected in all } 21 \\
\text { cultivars, } 19-508 \mu \mathrm{g} / \mathrm{g} \text { fw; } \\
E \text {-piceid: detected in } 19 \text { cultivars, } \\
\text { from n.d. to } 551 \mu \mathrm{g} / \mathrm{g} \text { fw; } \\
Z \text {-piceid: detected in } 20 \text { cultivars, } \\
\text { from n.d. to } 1196 \mu \mathrm{g} / \mathrm{g} \text { fw; } \\
E \text {-piceatannol: detected in } 7 \\
\text { cultivars, from n.d. to } 72.1 \mu \mathrm{g} / \mathrm{g} \text { fw; } \\
Z \text {-resveratrol: detected in the berry } \\
\text { skins at very low concentrations. }\end{array}$ & $\begin{array}{l}\text { Vincenzi } \\
\text { et al. } \\
\text { (2013) }\end{array}$ \\
\hline
\end{tabular}


Table 1 continued

\begin{tabular}{|c|c|c|c|c|c|}
\hline No. & Sample & Preparation conditions & $\begin{array}{l}\text { Analytical } \\
\text { method }\end{array}$ & Stilbenes: concentration $(\mu \mathrm{g} / \mathrm{g})$ & References \\
\hline 24 & $\begin{array}{l}\text { Red grapes } \\
\text { (Vitis } \\
\quad \text { vinifera) } \\
\text { Cultivars: } \\
\text { Raboso } \\
\text { Piave } \\
\text { Primitivo }\end{array}$ & $\begin{array}{l}\text { 1. Material: fresh } \\
\text { berries } \\
\text { 2. Solvent: } \mathrm{MeOH} \\
\text { 3. Conditions: room } \\
\text { temperature }\end{array}$ & $\begin{array}{l}\text { UHPLC- } \\
\text { QTOF- } \\
\text { MS }\end{array}$ & $\begin{array}{l}\text { Primitivo }(\mu \mathrm{g} / \mathrm{g} \text { fw }) \\
E \text {-astringin: } 0.8842 \pm 0.0034 \\
E \text {-piceid: } 2.3321 \pm 0.0489 \\
Z \text {-astringin: } 0.1214 \pm 0.0010 \\
\text { piceatannol: } 0.1815 \pm 0.0102 \\
Z \text {-piceid: } 1.7762 \pm 0.0474 \\
\text { Pallidol: } 0.3562 \pm 0.0026 \\
\text { Pallidol-3-O-glucoside: } 0.1875 \pm 0.0016 \\
\text { Parthenocissin A: } 0.1310 \pm 0.0011 \\
E \text {-resveratrol: } 1.1364 \pm 0.0536 \\
\text { Caraphenol B: } 0.1042 \pm 0.0014 \\
\text { Hopheapenol: } 0.1010 \pm 0.0023 \\
\text { Ampelopsin H/vaticanol C/isohopheaphenol: } \\
\text { 0.5366 } \pm 0.0129 \\
Z \text { - } \varepsilon \text {-viniferin: } 0.380 \pm 0.0070 \\
E \text { - } \varepsilon \text {-viniferin: } 0.7021 \pm 0.0074 \\
Z \text {-miyabenol C: } 0.2362 \pm 0.0078 \\
E \text {-miyabenol C: } 1.3578 \pm 0.0119 \\
\delta \text {-viniferins: } 0.0679 \pm 0.0011\end{array}$ & $\begin{array}{c}\text { Flamini } \\
\text { et al. } \\
\text { (2013) }\end{array}$ \\
\hline 25 & $\begin{array}{l}\text { Commercial } \\
\text { Grapes } \\
\text { (Vitis } \\
\quad \text { vinifera) } \\
\text { Cultivar: } \\
\text { Mencía }\end{array}$ & $\begin{array}{l}\text { 1. Material: } \\
\text { lyophilised skin } \\
\text { 2. Solvent: } \mathrm{MeOH} / \mathrm{HCl} \\
\text { (99:1 and 95:5) } \\
\text { 3. Conditions: room } \\
\text { temperature }\end{array}$ & $\begin{array}{l}\text { HPLC- } \\
\text { DAD }\end{array}$ & $\begin{array}{l}E \text {-resveratrol: } \\
2.10-3.28 \mu \mathrm{g} / \mathrm{g} \mathrm{dw}\end{array}$ & $\begin{array}{l}\text { Vilanova } \\
\text { et al. } \\
\text { (2015) }\end{array}$ \\
\hline 26 & $\begin{array}{l}\text { Grapes } \\
\text { (Vitis } \\
\quad \text { vinifera) } \\
\text { Cultivar: } \\
\text { Negro } \\
\quad \text { Amaro }\end{array}$ & $\begin{array}{l}\text { 1. Material: fresh } \\
\text { berries } \\
\text { 2. Solvent: } \mathrm{MeOH} \\
\text { 3. Conditions: room } \\
\text { temperature }\end{array}$ & $\begin{array}{l}\text { UHPLC- } \\
\text { QTOF- } \\
\text { MS }\end{array}$ & $\begin{array}{l}\text { Non-infected, infected grapes }(\mu \mathrm{g} / \mathrm{g} \mathrm{fw}) \\
\text { E-resveratrol: } 1.83 \pm 0.15,3.89 \pm 0.92 \\
\text { Piceatannol: } 0.65 \pm 0.08,0.78 \pm 0.30 \\
Z \text {-piceid: } 0.78 \pm 0.57,0.71 \pm 0.27 \\
\text { E-piceid: } 1.71 \pm 0.23,0.92 \pm 0.23 \\
\text { E-astringin: } 1.08 \pm 0.01,0.50 \pm 0.09 \\
Z \text {-astringin: } 0.05 \pm 0.01,0.05 \pm 0.01 \\
\text { Pallidol: } 0.70 \pm 0.09,0.59 \pm 0.25 \\
Z \text {-E-viniferin: } 0.29 \pm 0.05,0.25 \pm 0.02 \\
\omega \text {-viniferin: } 0.88 \pm 0.23,1.99 \pm 0.21 \\
Z \text {-miyabenol C: } 0.04 \pm 0.00,0.05 \pm 0.02 \\
\text { E-miyabenol C: } 0.66 \pm 0.07,1.46 \pm 0.60 \\
\text { Ampelopsin } \mathrm{H} / \mathrm{vaticanol} \mathrm{C} / \mathrm{hopeaphenol} / \\
\text { isohopeaphenol/vitisin } \mathrm{A} / \mathrm{B} / \mathrm{C}: 0.21 \pm 0.02, \\
0.57 \pm 0.02\end{array}$ & $\begin{array}{c}\text { Flamini } \\
\text { et al. } \\
\text { (2016) }\end{array}$ \\
\hline
\end{tabular}


Table 1 continued

\begin{tabular}{|c|c|c|c|c|c|}
\hline No. & Sample & Preparation conditions & $\begin{array}{l}\text { Analytical } \\
\text { method }\end{array}$ & Stilbenes: concentration $(\mu \mathrm{g} / \mathrm{g})$ & References \\
\hline 27 & $\begin{array}{l}\text { Grapes } \\
\text { (Vitis } \\
\quad \text { vinifera) } \\
\text { Cultivars: } \\
\text { Corvina } \\
\text { Raboso Piave }\end{array}$ & $\begin{array}{l}\text { 1. Material: fresh berries } \\
\text { 2. Solvent: } \mathrm{MeOH} \\
\text { 3. Conditions: room temperature }\end{array}$ & $\begin{array}{l}\text { UHPLC- } \\
\text { QTOF-MS }\end{array}$ & $\begin{array}{l}\text { Raboso Piave: fresh, dried }(\mu \mathrm{g} / \mathrm{g}) \\
\alpha \text {-viniferin: } 0.79 \pm 0.20,1.05 \pm 0.27 \\
\text { Z-piceid: } 4.00 \pm 1.32,5.34 \pm 1.76 \\
\text { E-astringin: } 1.44 \pm 0.30,1.91 \pm 0.39 \\
\text { Pallidol-3-O-glucoside: } 0.91 \pm 0.26 \\
1.21 \pm 0.34 \\
\text { Piceatannol: } 1.95 \pm 0.14, \\
2.59 \pm 0.19 \\
\text { Pallidol: } 1.02 \pm 0.15,1.36 \pm 0.21 \\
\text { Parthenocissin A: } 0.56 .0 \pm 0.05, \\
0.57 \pm 0.07 \\
Z \text { - } \varepsilon \text {-viniferin: } 1.55 \pm 0.22, \\
2.07 \pm 0.30 \\
E \text { - } \varepsilon \text {-viniferin: } 5.80 \pm 1.88, \\
7.73 \pm 2.50 \\
\delta \text {-viniferins: } 0.81 \pm 0.30, \\
1.08 \pm 0.40 \\
Z \text {-miyabenol C: } 0.61 \pm 0.19, \\
0.82 \pm 0.25 \\
E \text {-miyabenol C: } 5.51 \pm 0.87, \\
7.35 \pm 1.16 \\
E \text {-piceid: } 3.84 \pm 1.00,5.13 \pm 1.33 \\
E \text {-resveratrol: } 4.93 \pm 0.36, \\
6.58 \pm 0.48 \\
Z \text {-astringin: } 0.13 \pm 0.03,0.17 \pm 0.04\end{array}$ & $\begin{array}{l}\text { De Rosso et al. } \\
\text { ( 2016) }\end{array}$ \\
\hline 28 & $\begin{array}{l}\text { Grapes } \\
\text { (Vitis } \\
\quad \text { vinifera L.) } \\
\text { Cultivar: } \\
\text { Crimson } \\
\quad \text { Seedless }\end{array}$ & $\begin{array}{l}\text { 1. Material: freeze-dried fresh } \\
\text { grape skins } \\
\text { 2. Solvent: } \mathrm{Et}_{2} \mathrm{O} \\
\text { 3. Conditions: room temperature }\end{array}$ & HPLC-DAD & $\begin{array}{l}\text { After } U V \text {-C treatment }(\mu \mathrm{g} / \mathrm{g} \mathrm{fw}) \\
\text { E-resveratrol: } 121.62 \\
\text { Z-piceid: } 61.22 \\
\text { E-piceid: } 15.81 \\
\text { E-piceatannol: } 14.7 \\
\varepsilon \text {-viniferin: } 11.25 \\
\omega \text {-viniferin: } 5.8 \\
\text { Isohopeaphenol: } 4.27 \\
\text { Stilbenoid: } 234.67\end{array}$ & $\begin{array}{l}\text { Guerrero et al. } \\
\text { (2016) }\end{array}$ \\
\hline
\end{tabular}


Table 1 continued

\begin{tabular}{|c|c|c|c|c|c|}
\hline No. & Sample & Preparation conditions & $\begin{array}{l}\text { Analytical } \\
\text { method }\end{array}$ & Stilbenes: concentration $(\mu \mathrm{g} / \mathrm{g})$ & References \\
\hline 29 & $\begin{array}{l}\text { Grapes } \\
\text { (Vitis vinifera } \mathrm{L} . \text { ) } \\
28 \text { grape interspecific } \\
\text { hybrids } \\
2 \text { Vitis vinifera }\end{array}$ & $\begin{array}{l}\text { 1. Material: freeze-dried berries } \\
\text { 2. Solvent: } 80 \% \mathrm{MeOH} / 1 \% \mathrm{HCl} \\
\text { 3. Conditions: room temperature }\end{array}$ & $\begin{array}{l}\text { LC-PDA- } \\
\text { QTOF-MS } \\
\text { identification } \\
\text { UPLC-PDA- } \\
\text { FL } \\
\text { determination }\end{array}$ & $\begin{array}{l}\text { E-piceid: } \\
\text { Rielsing: } 71 \mu \mathrm{g} / \mathrm{g} \mathrm{dw} \\
\text { Hibernal: } 25 \mu \mathrm{g} / \mathrm{g} \mathrm{dw} \\
\text { Muscat Odeski: } \\
26 \mu \mathrm{g} / \mathrm{g} \mathrm{dw} \\
\text { Biona: } 19 \mu \mathrm{g} / \mathrm{g} \mathrm{dw}\end{array}$ & $\begin{array}{l}\text { Samoticha } \\
\text { et al. } \\
\text { (2017) }\end{array}$ \\
\hline 30 & $\begin{array}{l}\text { Wild-type plants of Vitis } \\
\text { amurensis Rupr. }\end{array}$ & $\begin{array}{l}\text { 1. Material: oven-dried fruits } \\
\text { 2. Solvent: } 96 \% \mathrm{EtOH} \\
\text { 3. Conditions: } 60{ }^{\circ} \mathrm{C} \\
\text { 4. Purification: } \mathrm{C} 18 \text { solid-phase } \\
\text { extraction }\end{array}$ & HPLC-MS & $\begin{array}{l}E \text {-piceid: } \\
55 \pm 12 \mu \mathrm{g} / \mathrm{g} \mathrm{dw} \\
Z \text {-piceid: } \\
127 \pm 41 \mu \mathrm{g} / \mathrm{g} \mathrm{dw} \\
E \text {-resveratrol: } \\
14 \pm 2 \mu \mathrm{g} / \mathrm{g} \mathrm{dw} \\
Z \text { - } \varepsilon \text {-viniferin: } \\
7 \pm 5 \mu \mathrm{g} / \mathrm{g} \mathrm{dw} \\
E \text { - } \varepsilon \text {-viniferin: } \\
46 \pm 5 \mu \mathrm{g} / \mathrm{g} \mathrm{dw} \\
E \text { - } \delta \text {-viniferin: nd }\end{array}$ & $\begin{array}{l}\text { Kiselev } \\
\text { et al. } \\
\text { (2017) }\end{array}$ \\
\hline 31 & $\begin{array}{l}\text { Lingonberry } \\
\text { (Vaccinium vitis-idaea L.) } \\
\text { Cultivar: } \\
\text { wild }\end{array}$ & $\begin{array}{l}\text { Method used for berries from } \\
\text { US location } \\
\text { 1. Material: lyophilized berries } \\
\text { 2. Solvent: } \mathrm{MeOH} / \text { acetone } / \mathrm{H}_{2} \mathrm{O} / \\
\mathrm{CH}_{3} \mathrm{COOH}(40: 40: 20: 0.1) \\
\text { 3. Conditions: } 40{ }^{\circ} \mathrm{C}, 1000 \mathrm{psi} \\
\text { 4. Purification: extraction with } \\
\text { EtOAc } \\
\text { 5. Derivatization: MSTFA/DFA/ } \\
\text { MeOH (3.5:1:0.5) } \\
\text { Method used for berries from } \\
\text { Canada } \\
\text { 1. Material: frozen berries } \\
\text { 2. Solvent: MeOH/acetone/ }{ }_{2} \mathrm{O} / \\
\text { HCOOH ( } 40: 40: 20: 0.1) \\
\text { 3. Conditions: room temperature } \\
\text { 4. Purification: C18 solid-phase } \\
\text { extraction } \\
\text { 5. Derivatization: MSTFA/DFA/ } \\
\text { MeOH (3.5:1:0.5) }\end{array}$ & GC-MS SIM & $\begin{array}{l}\text { Resveratrol: } \\
5.884 \mu \mathrm{g} / \mathrm{g} \mathrm{dw}\end{array}$ & $\begin{array}{l}\text { Rimando } \\
\text { and Cody } \\
(2005)\end{array}$ \\
\hline 32 & $\begin{array}{l}\text { Passion fruit } \\
\text { (Passiflora edulis) } \\
\text { seeds }\end{array}$ & $\begin{array}{l}\text { 1. Material: freeze-dried fruit } \\
\text { seeds } \\
\text { 2. Solvent: } 80 \% \mathrm{EtOH} \\
\text { 3. Conditions: room temperatrue }\end{array}$ & HPLC-UV & $\begin{array}{l}\text { Piceatannol: } \\
85400 \mu \mathrm{g} / \mathrm{g} \mathrm{dw} \\
\text { Scirpusin B: } \\
54700 \mu \mathrm{g} / \mathrm{g} \mathrm{dw}\end{array}$ & $\begin{array}{l}\text { Kawakami } \\
\text { et al. } \\
\text { (2014) }\end{array}$ \\
\hline
\end{tabular}


Table 1 continued

\begin{tabular}{|c|c|c|c|c|c|}
\hline No. & Sample & Preparation conditions & $\begin{array}{l}\text { Analytical } \\
\text { method }\end{array}$ & Stilbenes: concentration $(\mu \mathrm{g} / \mathrm{g})$ & References \\
\hline 33 & $\begin{array}{l}\text { Tomato } \\
\text { (Lycopersicon } \\
\text { esculentum Mill.) } \\
\text { Cultivars: } \\
\text { MicroTom } \\
\text { Beafsteak } \\
\text { UglyRipe } \\
\text { Heirloo } \\
\text { PlumTom }\end{array}$ & $\begin{array}{l}\text { 1. Material: lyophilized tomato } \\
\text { or grape skin } \\
\text { 2. Solvent: EtOAc } \\
\text { 3. Conditions: } 70^{\circ} \mathrm{C} \\
\text { 4. Derivatization: MSTFA/ } \\
\text { pyridine }\end{array}$ & GC-MS & $\begin{array}{l}\text { MicroTom } \\
\text { Z-resveratrol: } \\
2.71 \mu \mathrm{g} / \mathrm{g} \mathrm{dw} \\
\text { E-resveratrol: } \\
15.30 \mu \mathrm{g} / \mathrm{g} \mathrm{dw} \\
\text { Z-piceid: } 0.26 \mu \mathrm{g} / \mathrm{g} \mathrm{dw} \\
\text { E-piceid: } 0.10 \mu \mathrm{g} / \mathrm{g} \mathrm{dw} \\
\text { Beafsteak } \\
\text { nd } \\
\text { UglyRipe } \\
\text { E-resveratrol: } \\
\text { 0.38 } \mu \mathrm{g} / \mathrm{g} \mathrm{dw} \\
\text { Heirloom } \\
\text { Z-resveratrol: } \\
\text { 0.11 } \mu \mathrm{g} / \mathrm{g} \mathrm{dw} \\
\text { E-resveratrol: } \\
\text { 1.75 } \mu \mathrm{g} / \mathrm{g} \mathrm{dw} \\
\text { PlumTom } \\
\text { E-resveratrol: } \\
\text { 0.34 } \mu \mathrm{g} / \mathrm{g} \mathrm{dw}\end{array}$ & $\begin{array}{l}\text { Ragab et al. } \\
(2006)\end{array}$ \\
\hline
\end{tabular}

$n d$ not detected, $f w$ fresh weight, $d w$ dry weight

However, these studies only focused on berries at veraison and ripening. It is known that the biosynthesis and accumulation of stilbenes in berries after UV-C treatment depends on the developmental stages of fruits. From genomic analyses, it has been deduced that there are two main stages during grape development that are sensitive to UV-C irradiation (Pilati et al. 2007), namely, before and after veraison. "Before veraison" constitutes the restructuring phase of cell metabolism, characterized by an up-regulation of genes associated with hormone signalling and transcription. "After veraison" is characteristic of fruit ripening, whereas 'veraison' is characterized by an oxidative burst and antioxidant regulation. Therefore, it has been speculated that 'veraison' may be the most sensitive stage in which to apply UV-C treatment.

Under natural conditions, before veraison, the $E$ resveratrol content was very low in 'Beihong' ( $V$. vinifera $\times V$. amurensis) berries (Wang et al. 2015). From veraison to maturity, the $E$ - and $Z$-piceid contents increased. UV-C treatment significantly stimulated the biosynthesis of $E$-resveratrol and $E$ piceid. The response of berries to UV-C irradiation was also related to berry development. Among the six developmental stages, the stage at 55 DAA (days after anthesis, 2 weeks before veraison) was the most sensitive to UV-C treatment. The contents of $E$ resveratrol and $E$-piceid increased 292- and 11-fold, respectively (Table 2, no. 3). Along with developmental factors, the sensitivity of resveratrol synthesis to UV-C irradiation gradually declined, which may be associated with the regulation of STS by the Mybl4 promoter. STS expression was the highest when the berries were exposed to UV-C irradiation at 55 DAA, which may explain why resveratrol accumulated during this developmental stage. The expression of the Myb14 promoter reached maximum levels $12 \mathrm{~h}$ before STS. These results suggest that Myb14 expression may play an important role in the transcriptional regulation of resveratrol biosynthesis induced by UVC irradiation. 
Table 2 Influence of abiotic and biotic external stimuli on the presence of stilbenes in grapes

\begin{tabular}{|c|c|c|c|c|c|}
\hline No & Sample & External stimuli & Conditions & $\begin{array}{l}\text { Induction of stilbenes } \\
\text { content by fold increase } \\
(\uparrow) / \text { decrease }(\downarrow) \text { content }\end{array}$ & Ref. \\
\hline \multicolumn{6}{|c|}{ Abiotic preharvest treatments of grape } \\
\hline 1 & $\begin{array}{l}\text { Crimson } \\
\text { seedless table } \\
\text { grapes } \\
\text { (Vitis vinifera } \\
\text { L.) }\end{array}$ & UV-C treatment & $\begin{array}{l}\text { Light intensity: } 31.1 \mathrm{~W} / \mathrm{m}^{2} \\
\text { Dose: } 9330 \mathrm{~J} / \mathrm{m}^{2} \\
\text { Time: } 5 \mathrm{~min} \\
3 \text { days before harvest }\end{array}$ & $\begin{array}{l}E \text {-resveratrol: } 12 \uparrow \\
Z \text {-piceid: } 9 \uparrow \\
E \text {-piceid: } 5 \uparrow \\
E \text {-piceatannol: } 4 \uparrow \\
\varepsilon \text {-viniferin: } 7 \uparrow \\
\omega \text {-viniferin: } 4 \uparrow \\
\text { isohopeaphenol: } 3 \uparrow \\
\text { stilbenoids: } 4 \uparrow\end{array}$ & $\begin{array}{l}\text { (Guerrero } \\
\text { et al. 2016) }\end{array}$ \\
\hline 2 & $\begin{array}{l}\text { Redglobe } \\
\text { table grapes } \\
\text { (Vitis vinifera } \\
\text { L.) }\end{array}$ & UV-C treatment & $\begin{array}{l}\text { Treatment } 5 \text { day before harvest } \\
\text { Treatment } 3 \text { day before harvest } \\
\text { Treatment } 1 \text { day before harvest } \\
\text { Power: } 1040 \mathrm{~W} \text {, time: } 1 \mathrm{~min} \\
\text { Power: } 1040 \mathrm{~W} \text {, time: } 5 \mathrm{~min} \\
\text { Power: } 1040 \mathrm{~W} \text {, time: } 10 \mathrm{~min} \\
\text { Postharvest storage: } 3 \text { days, at } 20^{\circ} \mathrm{C}, 80 \% \mathrm{RH} \\
\text { Postharvest storage: } 3 \text { days, at } 4^{\circ} \mathrm{C}, 60 \% \mathrm{RH}\end{array}$ & $\begin{array}{l}E \text {-resveratrol: } 22 \uparrow \\
E \text {-resveratrol: } 46 \uparrow \\
E \text {-resveratrol: } 26 \uparrow \\
\varepsilon \text {-viniferin: } 10 \uparrow \\
\varepsilon \text {-viniferin: } 30 \uparrow \\
\varepsilon \text {-viniferin: } 20 \uparrow \\
E \text {-resveratrol: } 14.4 \uparrow \\
\varepsilon \text {-viniferin: } 3.4 \uparrow \\
E \text {-resveratrol: } 4 \uparrow \\
\varepsilon \text {-viniferin: } 0.3 \uparrow\end{array}$ & $\begin{array}{l}\text { (Guerrero } \\
\text { et al. 2015) }\end{array}$ \\
\hline 3 & $\begin{array}{l}\text { Beihong } \\
\text { grapes } \\
(\text { Vitis } \\
\text { vinifera } \times \text { Viti } \\
\text { s amurensis })\end{array}$ & $\begin{array}{l}\text { UV-C treatment } \\
\text { at different development stages (days } \\
\text { after anthesis - DAA) }\end{array}$ & $\begin{array}{l}55 \text { DAA } \\
126 \text { DAA } \\
\text { Illumination power: } 6 \mathrm{~W} / \mathrm{m}^{2} \\
\text { Distance: } 15 \mathrm{~cm} \\
\text { Time: } 10 \mathrm{~min} \\
\text { Temperature: } 25^{\circ} \mathrm{C} \text { in the dark } \\
\text { Storage conditions: at } 25^{\circ} \mathrm{C} \text { in the dark, time: } 0-72 \mathrm{~h}\end{array}$ & $\begin{array}{l}E \text {-resveratrol: } 292 \uparrow \\
E \text {-piceid: } 11 \uparrow \\
E \text {-resveratrol: } 6.9 \uparrow \\
E \text {-piceid: nd } \uparrow\end{array}$ & $\begin{array}{l}\text { (Wang et } \\
\text { al. 2015) }\end{array}$ \\
\hline \multicolumn{6}{|c|}{ Abiotic postharvest treatments of grape } \\
\hline 4 & $\begin{array}{l}\text { Grape varieties: } \\
3 \text { Vitis vinifera } \\
\text { sylvestris, } \\
7 \text { Vitis vinifera } \\
\text { sativa, } \\
2 \text { Hybrid Direct } \\
\text { Producers }\end{array}$ & UV-C treatment & $\begin{array}{l}\text { Power: } 500 \mathrm{~W} \\
\text { Distance: } 42 \mathrm{~cm} \\
\text { Time: } 60 \mathrm{~s} \\
\text { Light intensity: } 14.72 \mathrm{~mW} / \mathrm{cm}^{2} \\
\text { Storage conditions: } \\
\text { at } 18^{\circ} \mathrm{C}, 75 \% \mathrm{RH} \text { for } 7 \text { days }\end{array}$ & $\begin{array}{l}\text { Merlot: } \\
\text {-resveratrol: } 2.5 \uparrow \\
\text { piceatannol: } 4.3 \uparrow \\
\text { viniferins: } 7.2 \uparrow \\
\text { Syrah: } \\
E \text {-resveratrol: } 4.5 \uparrow \\
\text { piceatannol: } 0.5 \downarrow \\
\text { viniferins: nd } \uparrow \\
\text { Tempranillo: } \\
E \text {-resveratrol: } 11.2 \uparrow \\
\text { piceatannol: nd } \uparrow \\
\text { viniferins: } 7.5 \uparrow\end{array}$ & $\begin{array}{l}\text { (Guerrero } \\
\text { et al. } \\
2010 \mathrm{a})\end{array}$ \\
\hline 5 & $\begin{array}{l}\text { White/black } \\
\text { grapes } \\
\text { (Vitis vinifera } \\
\text { L.) }\end{array}$ & UV-C treatment & $\begin{array}{l}\text { Time: } 10 \mathrm{~min} \\
\text { Dose: } 0.36 \mathrm{~J} / \mathrm{cm} \\
\text { Storage time: } 48 \mathrm{~h}\end{array}$ & $\begin{array}{l}\text { Gamay: } \\
E \text {-piceid: } 0.3 \downarrow \\
Z \text {-piceid: } 0.1 \uparrow \\
E \text {-resveratrol: } 19 \uparrow \\
\varepsilon \text {-viniferin: } 2.6 \uparrow \\
\text { pterostilbene: } 0.5 \uparrow \\
\text { Chardonnay: } \\
E \text {-piceid: } 0.1 \downarrow \\
Z \text {-piceid: } 0.4 \downarrow \\
E \text {-resveratrol: } 1.2 \uparrow \\
\varepsilon \text {-viniferin: } 4.1 \uparrow \\
\text { pterostilbene: } 100 \uparrow\end{array}$ & $\begin{array}{l}\text { (Adrian et } \\
\text { al. 2000) }\end{array}$ \\
\hline 6 & $\begin{array}{l}\text { Napoleon table } \\
\text { grapes } \\
\text { (Vitis vinifera } \\
\text { L.) }\end{array}$ & UV-C treatment & $\begin{array}{l}\text { Distance: } 20-60 \mathrm{~cm} \\
\text { Temperature: } 25^{\circ} \mathrm{C} \\
\text { Treatment time and power: } \\
5 \mathrm{~s}, 30 \mathrm{~W} \\
5 \mathrm{~s}, 90 \mathrm{~W} \\
5 \mathrm{~s}, 240 \mathrm{~W} \\
5 \mathrm{~s}, 510 \mathrm{~W} \\
30 \mathrm{~s}, 30 \mathrm{~W} \\
30 \mathrm{~s}, 90 \mathrm{~W} \\
30 \mathrm{~s}, 240 \mathrm{~W} \\
60 \mathrm{~s}, 510 \mathrm{~W} \\
60 \mathrm{~s}, 30 \mathrm{~W}\end{array}$ & $\begin{array}{l}\text { Resveratrol: } \\
0.2 \uparrow \\
1.5 \uparrow \\
3.5 \uparrow \\
8.4 \uparrow \\
0.6 \uparrow \\
8 \uparrow \\
9.2 \uparrow \\
10.5 \uparrow \\
3.4 \uparrow \\
9.4 \uparrow\end{array}$ & $\begin{array}{c}\text { (Cantos et } \\
\text { al. 2001) }\end{array}$ \\
\hline
\end{tabular}


Table 2 continued

\begin{tabular}{|c|c|c|c|c|c|}
\hline & & & $\begin{array}{l}60 \mathrm{~s}, 90 \mathrm{~W} \\
60 \mathrm{~s}, 240 \mathrm{~W} \\
60 \mathrm{~s}, 510 \mathrm{~W} \\
300 \mathrm{~s}, 30 \mathrm{~W} \\
300 \mathrm{~s}, 90 \mathrm{~W} \\
300 \mathrm{~s}, 240 \mathrm{~W} \\
300 \mathrm{~s}, 510 \mathrm{~W} \\
\end{array}$ & $\begin{array}{l}10 \uparrow \\
10.5 \uparrow \\
5 \uparrow \\
9.4 \uparrow \\
8 \uparrow \\
6.5 \uparrow\end{array}$ & \\
\hline 7 & $\begin{array}{l}\text { Redglobe table } \\
\text { grape } \\
\text { (Vitis vinifera } \\
\text { L.) }\end{array}$ & UV-C treatment & $\begin{array}{l}\text { Dose: } 0.8,2.4,4.1 \mathrm{~kJ} / \mathrm{m}^{2} \\
\text { Distance: } 40 \mathrm{~cm} \\
\text { Treatment time: } 3 \mathrm{~min} \text {, storage time: } 48 \mathrm{~h} \text { at } 4^{\circ} \mathrm{C} \\
\text { Treatment time: } 3 \mathrm{~min} \text {, storage time: } 48 \mathrm{~h} \text { at } 25^{\circ} \mathrm{C}\end{array}$ & $\begin{array}{l}E \text {-piceid: } 1.2 \uparrow \\
Z \text {-piceid: } 2.6 \uparrow \\
E \text {-piceid: } 0.1 \uparrow \\
Z \text {-piceid: } 0.5 \uparrow \\
\end{array}$ & $\begin{array}{l}\text { (Crupi et } \\
\text { al. 2013) }\end{array}$ \\
\hline 8 & $\begin{array}{l}\text { Red grapes } \\
\text { (Vitis vinifera } \\
\text { L.): } \\
\text { Syrah, Merlot, } \\
\text { Cabernet } \\
\text { sauvignon, } \\
\text { Pinot noir }\end{array}$ & UV-C treatment & $\begin{array}{l}\text { Power: } 1020 \mathrm{~W} \\
\text { Distance: } 42 \mathrm{~cm} \\
\text { Time: } 60 \mathrm{~s} \\
\text { Storage conditions: } \\
\text { at } 20^{\circ} \mathrm{C} \text { for } 7 \text { days, } 80 \% \mathrm{RH}\end{array}$ & $\begin{array}{l}\text { Syrah, terroir Jerez: } \\
\text { resveratrol: } 2.7 \uparrow \\
\text { piceatannol: } 6.1 \uparrow \\
\text { viniferin: } 1.6 \uparrow \\
\text { total stilbenes: } 3.1 \uparrow \\
\text { Syrah, terroir Cabra: } \\
\text { resveratrol: } 1.1 \uparrow \\
\text { piceatannol: } 3.5 \uparrow \\
\text { viniferin: } 2 \uparrow \\
\text { total stilbenes: } 1.5 \uparrow\end{array}$ & $\begin{array}{l}\text { (Fernández } \\
\text {-Marín et } \\
\text { al. 2013) }\end{array}$ \\
\hline 9 & $\begin{array}{l}\text { Pinot Noir } \\
\text { (Vitis vinifera } \\
\text { L.) }\end{array}$ & UV-C treatment & $\begin{array}{l}\text { Distance: } 50 \mathrm{~cm} \\
\text { Light intensity: } 0.25 \mu \mathrm{W} / \mathrm{cm}^{2} \\
\text { Time: } 1 \mathrm{~h} \\
\text { Storage conditions: } \\
23 \mathrm{~h} \text { in the dark at } 25^{\circ} \mathrm{C}\end{array}$ & $\begin{array}{l}E \text {-resveratrol: } 355 \uparrow \\
\varepsilon \text {-viniferin: } 4.8 \uparrow \\
\text { piceid: } 2.4 \uparrow \\
Z \text {-resveratrol: } 0.7 \uparrow\end{array}$ & $\begin{array}{l}\text { (Suzuki et } \\
\text { al. 2015) }\end{array}$ \\
\hline 10 & $\begin{array}{l}\text { Red Globe } \\
\text { grapes } \\
\text { (Vitis vinifera } \\
\text { L.) }\end{array}$ & $\begin{array}{l}\text { UV treatment } \\
302.1 \mathrm{~nm} \text { resonant wavelength }(\mathrm{R}) \\
300.0 \mathrm{~nm} \text { non-resonant wavelength } \\
(\mathrm{NR})\end{array}$ & $\begin{array}{l}\text { Storage conditions: } 4^{\circ} \mathrm{C} \text { during } 4 \text { weeks } \\
\text { Treatment time: } 30 \text { min: } \mathrm{R} \\
\text { Treatment time: } 30 \text { min: } \mathrm{NR} \\
\text { Treatment time: } 45 \text { min: } \mathrm{R} \\
\text { Treatment time: } 45 \text { min: } \mathrm{NR} \\
\text { Treatment time: } 60 \text { min: } \mathrm{R} \\
\text { Treatment time: } 60 \text { min: } \mathrm{NR}\end{array}$ & $\begin{array}{l}E \text {-resveratrol: } \\
0.8 \uparrow \\
0.3 \downarrow \\
6.2 \uparrow \\
0.1 \uparrow \\
0.4 \downarrow \\
=\end{array}$ & $\begin{array}{l}\text { (Jiménez } \\
\text { Sánchez et } \\
\text { al. 2007) }\end{array}$ \\
\hline 11 & $\begin{array}{l}\text { Crimson red } \\
\text { table grapes } \\
\text { (Vitis labrusca) }\end{array}$ & $\begin{array}{l}\text { UV-C treatment and } 0.5 \% / 1 \% \\
\text { chitosan coating }(\mathrm{CHT})\end{array}$ & $\begin{array}{l}\text { Light intensity: } 2.82 \mathrm{~mW} / \mathrm{cm}^{2} \\
\text { Distance: } 60 \mathrm{~cm} \\
\text { Temperature: } 10^{\circ} \mathrm{C} \\
\text { Treatment with UV-C: } \\
\text { Treatment with UV-C and storage } 20^{\circ} \mathrm{C} / 24 \mathrm{~h} \text { : } \\
\text { Treatment with CHT } 0.5 \% \text { : } \\
\text { Treatment with UV-C, CHT } 0.5 \% \text {, storage } 5 \text { days: } \\
\text { Treatment with UV-C, CHT } 0.5 \% \text {, storage } 20^{\circ} \mathrm{C} / 24 \mathrm{~h}, 5 \\
\text { days: } \\
\text { Treatment with UV-C, CHT } 0.5 \% \text {, storage } 8 \text { days: } \\
\text { Treatment with UV-C, CHT } 0.5 \% \text {, storage } 20^{\circ} \mathrm{C} / 24 \mathrm{~h}, 8 \\
\text { days: }\end{array}$ & $\begin{array}{l}E \text {-resveratrol: } \\
0.4 \uparrow \\
3 \uparrow \\
= \\
2 \uparrow \\
4.9 \uparrow \\
2.9 \uparrow \\
3.7 \uparrow\end{array}$ & $\begin{array}{l}\text { (Freitas et } \\
\text { al. 2015) }\end{array}$ \\
\hline 12 & $\begin{array}{l}\text { Beihong } \\
(\text { Vitis } \\
\text { vinifera } \times V \text {. } \\
\text { amurensis }) \\
\text { Hongbaladuo }\end{array}$ & Treatment with $\mathrm{UV}$ and $\mathrm{CaCl}_{2}$ & $\begin{array}{l}\text { Light intensity: } 6 \mathrm{~W} / \mathrm{m}^{2} \\
\text { Distance: } 15 \mathrm{~cm} \\
\text { Time: } 10 \mathrm{~min} \\
\text { Storage conditions: } \\
\text { at } 25^{\circ} \mathrm{C} \text { in the dark for } 24 \mathrm{~h} \text {, then at }-1^{\circ} \mathrm{C} \text { for } 27 \text { days, } \mathrm{RH} \\
95^{\%} \\
\text { Treatment with } \mathrm{CaCl}_{2} \text { : } \\
\text { Treatment with UV-C: } \\
\text { Treatment with UV-C and } \mathrm{CaCl}_{2} \text { : }\end{array}$ & $\begin{array}{l}E \text {-resveratrol: } 0.5 \uparrow \\
E \text {-piceid: }= \\
Z \text {-piceid: } 0.2 \downarrow \\
E \text {-resveratrol: } 11 \uparrow \\
E \text {-piceid: } 0.8 \uparrow \\
Z \text {-piceid: } 0.6 \downarrow \\
E \text {-resveratrol: } 16.7 \uparrow \\
E \text {-piceid: } 0.6 \uparrow \\
Z \text {-piceid: } 1.4 \downarrow\end{array}$ & $\begin{array}{l}\text { (Wang et } \\
\text { al. 2013) }\end{array}$ \\
\hline 13 & $\begin{array}{l}\text { Campbell Early } \\
\text { grapes } \\
(\text { Vitis labrusca } \\
\times \text { V. vinifera) }\end{array}$ & Ultrasonication treatment $(40 \mathrm{kHz})$ & $\begin{array}{l}\text { Temperature storage: } 25^{\circ} \mathrm{C} \\
\text { Treatment time } 5 \mathrm{~min}: \\
\text { Treatment time } 10 \mathrm{~min}: \\
\text { Treatment time } 15 \mathrm{~min}: \\
\text { Treatment time } 5 \mathrm{~min}, 6 \mathrm{~h} \text { storage: } \\
\text { Treatment time } 10 \mathrm{~min}, 6 \mathrm{~h} \text { storage: } \\
\text { Treatment time } 15 \mathrm{~min}, 6 \text { h storage: } \\
\text { Treatment time } 5 \mathrm{~min}, 12 \text { h storage: }\end{array}$ & $\begin{array}{l}\text { E-resveratrol: } \\
1.3 \uparrow \\
1.3 \uparrow \\
0.8 \uparrow \\
6.7 \uparrow \\
0.3 \uparrow \\
=\end{array}$ & $\begin{array}{c}\text { (Hasan and } \\
\text { Baek } \\
\text { 2013) }\end{array}$ \\
\hline
\end{tabular}


Table 2 continued

\begin{tabular}{|c|c|c|c|c|c|}
\hline & & & $\begin{array}{l}\text { Treatment time } 10 \mathrm{~min}, 12 \mathrm{~h} \text { storage: } \\
\text { Treatment time } 15 \mathrm{~min}, 12 \mathrm{~h} \text { storage: }\end{array}$ & $\begin{array}{l}2 \uparrow \\
0.2 \uparrow \\
0.3 \uparrow\end{array}$ & \\
\hline 14 & $\begin{array}{l}\text { Superior } \\
\text { Seedless } \\
\text { (Vitis } \\
\text { vinifera } \text { L.) } \\
\text { Regina Victoria } \\
(\text { Vitis } \\
\text { vinifera } \text { L.) } \\
\text { Cardinal CL80 } \\
(\text { Vitis } \\
\text { vinifera } \text { L. })\end{array}$ & Ozone treatment & $\begin{array}{l}\frac{\text { Storage conditions: }}{5^{\circ} \mathrm{C} \text { for } 0,15,30,56,72 \text { days }} \\
\text { Air, continuous } 2 \mathrm{ppm} \mathrm{O}_{3} \text { : } \\
\text { Air, intermittent } 2 \mathrm{ppm} \mathrm{O}_{3}, 12 \mathrm{~h} \text { /day: } \\
\text { Air, continuous } 2 \mathrm{ppm} \mathrm{O}_{3} \text { : } \\
\text { Air, intermittent } 2 \mathrm{ppm} \mathrm{O}_{3}, 12 \mathrm{~h} \text { /day: } \\
\text { Air, continuous } 2 \mathrm{ppm} \mathrm{O}_{3} \text { : } \\
\text { Air, intermittent } 2 \mathrm{ppm} \mathrm{O}_{3}, 12 \mathrm{~h} \text { /day: }\end{array}$ & $\begin{array}{l}\text { Resveratrol: } \\
\text { Superior Seedless } \\
0.3 \downarrow \\
0.2 \uparrow \\
\underline{\text { Cardinal CL80 }} \\
= \\
0.5 \uparrow \\
\underline{\text { Regina Victoria }} \\
1 \downarrow \\
0.6 \uparrow\end{array}$ & $\begin{array}{l}\text { (Cayuela et } \\
\text { al. 2010) }\end{array}$ \\
\hline 15 & $\begin{array}{l}\text { Table grape } \\
\text { Napoleon } \\
(\text { Vitis } \\
\text { vinifera } \mathrm{L} .)\end{array}$ & Ozone treatment & $\begin{array}{l}8 \mathrm{ppm} \mathrm{O}_{3} \text {, storage } 38 \text { days at } 0^{\circ} \mathrm{C} \text { : } \\
8 \text { ppm } \mathrm{O}_{3} \text {, storage } 6 \text { days at } 15^{\circ} \mathrm{C} \text { : }\end{array}$ & $\begin{array}{l}E \text {-piceid: } 11 \uparrow \\
E \text {-resveratrol: } 2.5 \uparrow \\
\text { stilbenoids: } 0.6 \uparrow \\
E \text {-piceid: } 4.5 \uparrow \\
E \text {-resveratrol: } 3.1 \uparrow \\
\text { stilbenoids: } 3.6 \uparrow\end{array}$ & $\begin{array}{l}\text { (Artés- } \\
\text { Hernández } \\
\text { et al. 2003) }\end{array}$ \\
\hline 16 & $\begin{array}{l}\text { Campbell Early } \\
\text { (Vitis labrusca) } \\
\text { Kyoho grapes } \\
\text { (Vitis } \\
\text { labruscana } \\
\text { Bailey) }\end{array}$ & $\begin{array}{l}\text { Treatments with: } \\
\text { white fluorescent light }(\mathrm{FL}) \\
380 \mathrm{~nm} \text { purple LED } \\
440 \mathrm{~nm} \text { blue LED } \\
660 \mathrm{~nm} \text { red LED }\end{array}$ & $\begin{array}{l}\text { Time: } 48 \mathrm{~h} \text {, at } 25^{\circ} \mathrm{C} \\
\text { Storage conditions: } 0-24 \mathrm{~h} \text { at } 25^{\circ} \mathrm{C} \\
\text { Treatment with FL: } \\
\text { Treatment with } 380 \mathrm{~nm} \text { : } \\
\text { Treatment with } 440 \mathrm{~nm}: \\
\text { Treatment with } 660 \mathrm{~nm} \text { : }\end{array}$ & $\begin{array}{l}\text { Campbell Early } \\
E \text {-resveratrol: } 1.8 \uparrow \\
Z \text {-resveratrol: }= \\
\text { piceatannol: } 0.6 \uparrow \\
E \text {-piceid: } 0.1 \uparrow \\
Z \text {-piceid: }= \\
\text { E-resveratrol: } 2.8 \uparrow \\
Z \text {-resveratrol: }= \\
\text { piceatannol: } 0.4 \uparrow \\
E \text {-piceid: } 0.4 \uparrow \\
Z \text {-piceid: }= \\
\text { E-resveratrol: } 7.4 \uparrow \\
Z \text {-resveratrol: }= \\
\text { Piceatannol: } 4.6 \uparrow \\
E \text {-piceid: } 1.1 \uparrow \\
Z \text {-piceid: } 3 \uparrow \\
E \text {-resveratrol: } 6.2 \uparrow \\
Z \text {-resveratrol: }= \\
\text { piceatannol: } 3 \uparrow \\
E \text {-piceid: } 0.5 \uparrow \\
Z \text {-piceid: }=\end{array}$ & $\begin{array}{l}\text { (Ahn et al. } \\
\text { 2015) }\end{array}$ \\
\hline 17 & $\begin{array}{l}\text { White grapes } \\
\text { (Vitis vinifera) } \\
\text { Aledo variety }\end{array}$ & Dry nitrogen treatment & Time: $24 \mathrm{~h}$ & $E$-resveratrol: $2 \uparrow$ & $\begin{array}{l}\text { (Jiménez et } \\
\text { al. 2007) }\end{array}$ \\
\hline 18 & $\begin{array}{l}\text { Corvina grapes } \\
\text { (Vitis vinifera } \\
\text { L.) } \\
\text { Raboso Piave } \\
\text { (Vitis vinifera } \\
\text { L.) }\end{array}$ & $\begin{array}{l}\text { Withering process (dehydration } \\
\text { process) }\end{array}$ & $18^{\circ} \mathrm{C}, 40 \% \mathrm{RH}, 30$ days and 60 days & 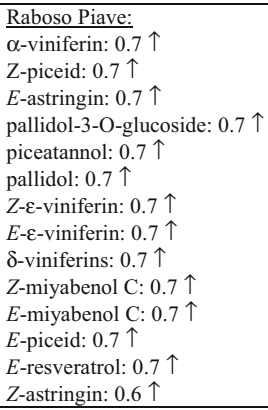 & $\begin{array}{l}\text { (De Rosso } \\
\text { et al. 2016) }\end{array}$ \\
\hline \multicolumn{6}{|c|}{ Combined abiotic pre- and postharvest treatments of grape } \\
\hline 19 & $\begin{array}{l}\text { Syrah red } \\
\text { grapes } \\
\text { (Vitis vinifera } \\
\text { L.) }\end{array}$ & $\begin{array}{l}\text { 1) Preharvest methyl jasmonate } \\
\text { (MEJA) treatment, } \\
\text { 2) Postharvest UV-C treatment, } \\
\text { 3) Preharvest methyl jasmonate/ } \\
\text { postharvest UV-C treatment (MEJA- } \\
\text { UV-C) }\end{array}$ & $\begin{array}{l}\text { Power: } 1020 \mathrm{~W} \\
\text { Distance: } 42 \mathrm{~cm} \\
\text { Time: } 60 \mathrm{~s} \\
\text { Storage conditions: at } 20^{\circ} \mathrm{C} \text { for } 4 \text { days, } 80 \% \mathrm{RH} \\
\text { 1) Preharvest treatment with MEJA: }\end{array}$ & $\begin{array}{l}\frac{2 \text { days after treatment: }}{E \text {-resveratrol: } 0.5 \uparrow} \\
\text { piceatannol: } 0.4 \uparrow \\
\text { isorphapontigenin: nd } \uparrow\end{array}$ & $\begin{array}{l}\text { (Fernández } \\
\text {-Marín et } \\
\text { al. 2014) }\end{array}$ \\
\hline
\end{tabular}


Table 2 continued

\begin{tabular}{|c|c|c|c|c|c|}
\hline & & & $\begin{array}{l}\text { 2) Postharvest treatment with UV-C: } \\
\text { 3) Treatment with MEJA (preharvest) and UV-C } \\
\text { (postharvest): }\end{array}$ & $\begin{array}{l}\varepsilon \text {-viniferin: nd } \\
4 \text { days after treatment: } \\
E \text {-resveratrol: } 0.5 \uparrow \\
\text { piceatannol: } 0.8 \uparrow \\
\text { isorphapontigenin: } 0.5 \uparrow \\
\varepsilon \text {-viniferin: } 1 \uparrow \\
\text { at harvest: } \\
E \text {-resveratrol: } 1 \uparrow \\
\text { piceatannol: nd } \uparrow \\
\text { isorphapontigenin: nd } \\
\varepsilon \text {-viniferin: nd } \\
2 \text { days after treatment: } \\
E \text {-resveratrol: }= \\
\text { piceatannol: }= \\
\text { isorphapontigenin: nd } \\
\varepsilon \text {-viniferin: nd } \\
4 \text { days after treatment: } \\
E \text {-resveratrol: } 0.5 \uparrow \\
\text { piceatannol: } 0.4 \uparrow \\
\text { isorphapontigenin: } 0.7 \uparrow \\
\varepsilon \text {-viniferin: } 0.4 \uparrow \\
2 \text { days after treatment: } \\
E \text {-resveratrol: } 0.6 \uparrow \\
\text { piceatannol: } 0.3 \uparrow \\
\text { isorphapontigenin: nd } \uparrow \\
\varepsilon \text {-viniferin: } n d \\
4 \text { days after treatment: } \\
E \text {-resveratrol: } 2 \uparrow \\
\text { piceatannol: } 1.7 \uparrow \\
\text { isorphapontigenin: } 1.8 \uparrow \\
\varepsilon \text {-viniferin: } 1.7 \uparrow\end{array}$ & \\
\hline 20 & $\begin{array}{l}\text { Autumn Black } \\
\text { grapes } \\
\text { (Vitis vinifera } \\
\text { L.) } \\
\text { B36-55 } \\
\text { (Vitis vinifera } \\
\text { L.) }\end{array}$ & $\begin{array}{l}\text { 1) Preharvest treatment with chitosan } \\
\text { 2) Postharvest treatment with UV-C } \\
\text { 3) Postharvest treatment with UV- } \\
\text { C/chitosan }\end{array}$ & $\begin{array}{l}\text { Dose: } 0.36 \mathrm{~J} / \mathrm{cm}^{2} \\
\text { Time: } 10 \mathrm{~min} \\
\text { Distance: } 10 \mathrm{~cm} \\
\text { Storage conditions: } 48 \mathrm{~h} \text { at } 20^{\circ} \mathrm{C}, \mathrm{RH} 95-98 \% \\
\text { Preharvest treatment with chitosan } \\
\text { Postharvest treatment with UV-C } \\
\text { Postharvest treatment with UV-C/chitosan }\end{array}$ & $\begin{array}{l}E \text {-resveratrol in B36-55: } \\
= \\
8.8 \uparrow \\
11 \uparrow\end{array}$ & $\begin{array}{l}\text { (Romanazz } \\
\text { i et al. } \\
2006)\end{array}$ \\
\hline \multicolumn{6}{|c|}{ Biotic postharvest treatments of grape } \\
\hline 21 & $\begin{array}{l}\text { Palomino } \\
\text { fino grapes } \\
\text { (Vitis vinifera } \\
\text { L.) }\end{array}$ & Infection with Botrytis cinerea & $\begin{array}{l}\text { Infection degree } 50 \% \text { : } \\
\text { Infection degree } 75 \% \text { : } \\
\text { Infection degree } 100 \% \text { : }\end{array}$ & $\begin{array}{l}\text { resveratrol: } 1.3 \downarrow \\
\text { piceid: } 1.3 \uparrow \\
\text { resveratrol: } 0.2 \uparrow \\
\text { piceid: } 2.6 \uparrow \\
\text { resveratrol: } 0.4 \uparrow \\
\text { piceid: } 3.5 \uparrow \\
\end{array}$ & $\begin{array}{l}\text { (Roldán et } \\
\text { al. 2003) }\end{array}$ \\
\hline 22 & $\begin{array}{l}\text { Negro Amaro } \\
\text { grapes } \\
\text { (Vitis vinifera } \\
\text { L.) }\end{array}$ & Infection with $A$. carbonarius & at $20^{\circ} \mathrm{C}$ for 5 days & 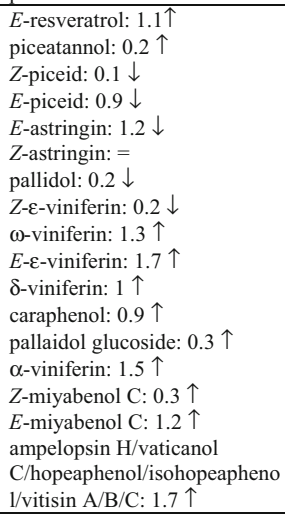 & $\begin{array}{l}\text { (Flamini et } \\
\text { al. 2016) }\end{array}$ \\
\hline \multicolumn{6}{|c|}{ Combined abiotic and biotic postharvest treatments of grape } \\
\hline 23 & $\begin{array}{l}\text { Napoleon } \\
\text { table grape } \\
\text { (Vitis vinifera } \\
\text { L.) }\end{array}$ & $\begin{array}{l}\text { UV-C treatment and fungal infection } \\
\text { with ochratoxigenic Aspergillus }\end{array}$ & $\begin{array}{l}\text { Illumination power: } 50 \mathrm{~W} / \mathrm{m}^{2} \\
\text { Distance: } 40 \mathrm{~cm} \\
\text { Time: } 60 \mathrm{~s} \\
\text { Temperature: } 15^{\circ} \mathrm{C} \\
\text { Storage condition: at } 22^{\circ} \mathrm{C} \\
\text { Undamaged grape: }\end{array}$ & & $\begin{array}{l}\text { (Selma et } \\
\text { al. 2008) }\end{array}$ \\
\hline
\end{tabular}


Table 2 continued

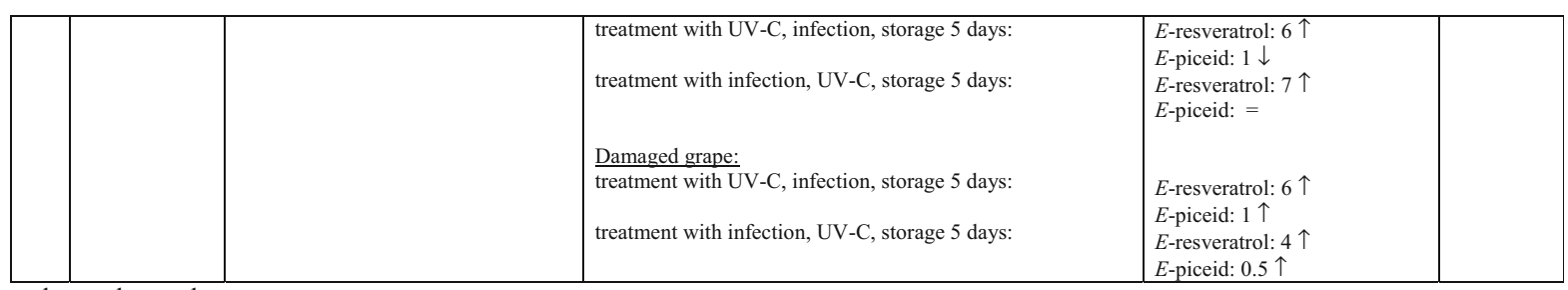

nd: not detected

$=$ no changes, $\uparrow$ increase, $\downarrow$ decrease

\section{Abiotic postharvest treatments of grape}

Postharvest treatment with UV-C light has been proposed as a valuable method to increase the stilbenes content in the grape berries (Langcake and Pryce 1977; Douillet-Breuil et al. 1999; Versari et al. 2001; Petit et al. 2009; Yin et al. 2016).

Postharvest treatment of grape varieties with UV-C resulted in higher concentrations of stilbenes, such as E-resveratrol, piceatannol, viniferins and pterostilbene (Table 2, no. 4, 5) (Adrian et al. 2000; Guerrero et al. 2010a). Differences in concentration after UV-C irradiation depended on the variety and campaign, but not on the grape subspecies. Each variety seemed to be influenced to a different degree by the climate. Thus, the same variety behaved in a different way in each campaign, and climate could determine the final concentration of stilbenes.

The highest accumulation of resveratrol (tenfold) in irradiated Napoleon grapes was achieved using the following combination of parameters: irradiation power, $510 \mathrm{~W}$; irradiation time, 30 or $60 \mathrm{~s}$; irradiation distance, $40 \mathrm{~cm}$; and elapsed days, 3 (Table 2, no. 6). Therefore, controlled UV irradiation parameters are useful as a simple postharvest treatment to increase the resveratrol concentration in Napoleon grapes (Cantos et al. 2001).

To achieve the highest possible stilbene accumulation, the interactive effects of storage time, temperature and UV-C irradiation on the stilbene content in postharvest Red globe table grapes were investigated (Table 2, no. 7) (Crupi et al. 2013). During storage, both cold storage and UV-C doses of 3 min raised the contents of $Z$ - and $E$-piceid, achieving 90 and $34 \mu \mathrm{g} / \mathrm{g}$ in skin, respectively, which was approximately threefold higher than those in control berry samples. Similar results were found in Napoleon table grapes (Cantos et al. 2001). Cold storage in combination with UV irradiation increased the piceid concentration more than cold storage alone. Also Cho et al. (2012) reported that it is possible to enrich resveratrol content in harvested grapes by modulating cell metabolism with UV treatment and storage conditions. Storage temperature had an effect on time-delayed resveratrol biosynthesis after removal of the UV irradiation. A larger amount of resveratrol was formed when UVtreated grapes were stored at higher temperature.

After UV-C postharvest irradiation, all of the red grape varieties in each terroir increased their resveratrol, piceatannol and viniferin contents (Table 2, no. 8) (Fernández-Marín et al. 2013). The stilbene content was different depending on the variety and the terroir. Cabra was the terroir where the varieties achieved the highest induction capacity $(2.02 \mu \mathrm{g} / \mathrm{g}$ per day after UV-C irradiation), especially the Syrah variety. This is in agreement with previous research in which Syrah increased its stilbene content more than the other thirteen varieties studied (Guerrero et al. 2010a). However, the highest increase in the resveratrol and piceatannol contents in the Syrah variety was from the Jerez terroir, which amounted to 2.7 and 6.1 times, respectively, in comparison to those in the untreated berries.

With regard to piceatannol and viniferins, higher concentrations were found in varieties that achieved higher resveratrol levels because resveratrol has been proposed as the precursor of the other stilbenes (Coutos-Thévenot et al. 2001). Thus, it could be 
concluded that resveratrol determines the tendency for the synthesis of other stilbenes and the amount of total stilbenes.

Moreover, varieties reached the highest level of resveratrol induction in different periods of time depending on the terroir. The day of maximum concentration $(\mathrm{dm})$ was constant for Cabernet sauvignon $(\mathrm{dm}=7)$. Pinot noir showed the same $\mathrm{dm}$ in all terroirs $(\mathrm{dm}=6)$, except for Cadiar, where it was delayed 1 day $(\mathrm{dm}=7)$. However, in Syrah and Merlot varieties, the $\mathrm{dm}$ changed depending on the terroir, ranging from 4 to 7 days.

Treatment of the postharvested Pinot Noir grape berries with UV-C irradiation increased the $E$-resveratrol, $\varepsilon$-viniferin, piceid and $Z$-resveratrol contents $355,4.8,2.4$ and 0.7 times, respectively, in comparison with those in the control sample (Table 2, no. 9) (Suzuki et al. 2015). Transcriptome analysis revealed that 238 genes were up-regulated more than fivefold in grape berry skin by UV-C treatment. Enrichment analysis of the gene ontology terms showed that genes encoding stilbene synthase were enriched in the upregulated genes.

Two different wavelengths were used for the UV treatment of red grapes: $302.1 \mathrm{~nm}$ for the resonant wavelength and $300.0 \mathrm{~nm}$ for the non-resonant wavelength (Table 2, no. 10) (Jiménez Sánchez et al. 2007). Four sets of irradiation times were selected for each of the two different wavelengths: 15, 30, 45 and $60 \mathrm{~min}$. The use of photons of resonant energy to produce absorption through the real electronic states of the molecule significantly increased the absorption yield, producing an important effect on the photoinduced $E$ resveratrol level in the grapes. The enhancement was optimal for $45 \mathrm{~min}$ of irradiation at $302.1 \mathrm{~nm}$. The samples were prepared for analysis immediately after irradiation to detect the direct effect of resonant elicitation.

The combination of UV-C treatment with a chitosan coating and incubation was investigated (Table 2, no. 11) (Freitas et al. 2015). The concentration of $E$-resveratrol in red table grapes with a $0.5 \%$ chitosan coating treated with UV-C irradiation, incubated at $20{ }^{\circ} \mathrm{C}$ and then stored for 5 days under refrigeration was approximately 5 and 2.5 times higher than that in control and UV-C treated grapes with a $0.5 \%$ chitosan coating, respectively. After 8 days of storage at $4{ }^{\circ} \mathrm{C}$, treated berries also showed a 2.9 times higher content of $E$-resveratrol and lower susceptibility to fungal decay than control grapes.

The individual and combined effects of $\mathrm{CaCl}_{2}$ and ultraviolet light on the biosynthesis of resveratrol in berry skins were investigated (Table 2, no. 12) (Wang et al. 2013). $\mathrm{CaCl}_{2}$ application had no effect on the $E$ piceid content and little influence on the Z-piceid content, but it resulted in a 0.5 times higher content of $E$-resveratrol compared with that in the control sample. UV-C and UV-C irradiation with $\mathrm{CaCl}_{2}$ treatment increased $E$-resveratrol and $E$-piceid biosynthesis and accumulation in berry skins. This accumulation continued until 13 days after cold storage. Then, the $E$-resveratrol content slightly decreased but still remained at high levels at the end of storage, while the $E$-piceid content increased continuously until the end of storage. In contrast, the $Z$-piceid content after UV-C and UV-C/ $\mathrm{CaCl}_{2}$ treatments decreased by 0.6 and 1.4 times, respectively, compared with that in the control sample.

$\mathrm{UV}-\mathrm{C}$ and $\mathrm{UV}-\mathrm{C} / \mathrm{CaCl}_{2}$ treatments significantly stimulated the expression of PAL, C4H, $4 C L$ and STS, which are related to the biosynthesis of $E$-resveratrol. Moreover, the expression levels of these genes in the $\mathrm{UV}-\mathrm{C} / \mathrm{CaCl}_{2}$ combination treatment were higher than those in the UV-C irradiation treatment. The expression of $P A L, C 4 H, 4 C L$, and STS in both treatments reached a maximal content at $12 \mathrm{~h}$ after initiating the treatment and then declined rapidly to approach the control level at the end of the experiment. $\mathrm{CaCl}_{2}$ treatment alone did not modify the expression of any of these genes (Wang et al. 2013).

Increased accumulation of $E$-resveratrol in grape skin by ultrasonication treatment has also been investigated (Table 2, no. 13) (Hasan and Baek 2013). A significantly higher amount of $E$-resveratrol over that in the control sample was observed following ultrasonication treatment. Ultrasonic treatment for $5 \mathrm{~min}$ followed by $6 \mathrm{~h}$ of incubation induced the highest levels of $E$-resveratrol, with amounted to a level that was 6.7 times higher than that in the control sample. However, the treatment did not lead to an increase in the maintenance of the level of $E$ resveratrol. When the amount of $E$-resveratrol was measured again in fruits incubated for another $6 \mathrm{~h}$, it had decreased drastically to the level in samples treated by $5 \mathrm{~min}$ of ultrasonication without any incubation. 
In grape skin, the expression levels of resveratrol synthase increased directly in response to $5 \mathrm{~min}$ of ultrasonication treatment and were then maintained during $12 \mathrm{~h}$ of incubation. This suggests that the accumulation of $E$-resveratrol in grape in response to ultrasonication occurs in a time-dependent manner based on the induction of the resveratrol synthase gene.

Ozone was also found to stimulate the synthesis of stilbenes in grape berries (Sarig et al. 1996; Palou et al. 2002). Continued and intermittent ozone treatments $(2 \mathrm{ppm})$ were applied during the storage of 3 varieties of table grapes at $5{ }^{\circ} \mathrm{C}$ for 72 days (Table 2, no. 14) (Cayuela et al. 2010). The continuous presence of ozone in the storage atmosphere inhibited the biosynthesis of resveratrol, whereas intermittent treatment with $\mathrm{O}_{3}$ stimulated its biosynthesis. It was found that the treatment consisting of intermittent shocks of $8 \mathrm{ppm} \mathrm{O}_{3}$ had the strongest influence on table grapes (Artés-Hernández et al. 2003). It was also found that the grapes stored in an atmosphere containing $0.1 \mathrm{ppm}$ $\mathrm{O}_{3}$ exhibited higher concentrations of resveratrol than those kept in air. Mild ozone treatments, such as those using ozonized water, which are effective in the control of microbial growth, did not induce stilbenoid accumulation in grapes (Gonzalez Ureña et al. 2003). Continuous exposure of the berries to a high concentration of $\mathrm{O}_{3}$ could reduce the content of antioxidant compounds such as resveratrol produced by the plant as a defensive metabolite against oxidative stress.

The influence of the combination of ozone and UVtreatment on stilbenes content was also investigated (González-Barrio et al. 2006). The results showed that UV-C was generally much more efficient (shorter treatment time) in inducing resveratrol content than ozone. However, with regard to total stilbenoids accumulated in the grape skin, the ozone treatment with the highest concentration and the longest time led to higher stilbenoid content than the UV-C irradiation. Also the viniferin content accumulated was threefold higher than that induced by the UV-C treatment. However, UV-C light treatment resulted in less damage to grape tissues than ozone gas.

Different gaseous treatments have been applied because of their efficacy in ensuring the quality of Napoleon table grapes (Table 2, no. 15) (ArtésHernández et al. 2003). The E-resveratrol content increased up to threefold its value sampled at harvest in $\mathrm{O}_{3}$ shock-treated clusters and up to twofold for other treatments, probably because these treatments induced a faster biosynthesis of this compound. The increases were more marked when clusters were stored at $15^{\circ} \mathrm{C}$, which is in agreement with previous reports, where control and UV-treated cv. Napoleon table grapes increased their resveratrol content after the grapes were transferred to $15^{\circ} \mathrm{C}$ (Cantos et al. 2000). However, $\mathrm{O}_{3}$-treated clusters that underwent shock treatment increased their $E$-piceid content by 11 and 4.5 times compared to that sampled at harvest.

The accumulation of stilbenes and the expression of genes related to their synthesis in 'Campbell Early' and 'Kyoho' grapes were investigated by irradiating the harvested grapes with four different light sources for $48 \mathrm{~h}$ (Table 2, no. 16) (Ahn et al. 2015). The total concentrations of five stilbene derivatives at $24 \mathrm{~h}$ after irradiation differed in response to different light sources and cultivars. The accumulation of stilbenes in the skins of two grape cultivars and the expression of PAL and STS1 genes were induced under mainly red and blue LED light. The amount of stilbenes tended to be higher in blue and red light-treated grapes than in the grape berries treated with white fluorescent or purple light. Among the stilbenes tested, $E$-resveratrol was present in 'Campbell Early' berries treated with blue, red, purple and fluorescent light at levels 7.4-, 6.2-, 2.8- and 1.8-fold higher than the levels in the control sample, respectively. The expression of $P A L$, CHS, CHI, STS1, STS12 and ROMT genes was differently induced in response to irradiation with different light sources in both grape cultivars. The mRNA levels of $P A L$ and STS1 were higher than those of CHS, CHI, STS12 and ROMT in the two grape berries. The results indicated that red and blue LEDs induced the accumulation of stilbenes and the expression of genes related to their syntheses in grape berries.

Accumulation of $E$-resveratrol in post-harvested grapes by dry nitrogen treatment was also investigated (Table 2, no. 17) (Jiménez et al. 2007). E-resveratrol content in the grape berries increased with the duration of the dry nitrogen treatment up to $24 \mathrm{~h}$ (twofold). For longer treatment, only a slight enhancement was observed. However, shorter treatments kept higher content of $E$-resveratrol during several days with no appreciable damage of the grape berries regarding to their organoleptic quality.

It was also observed that storage conditions influence the stilbene concentration (Table 2, no. 18). 
Grapes stored under mild conditions $\left(20^{\circ} \mathrm{C}\right)$ favoured $E$-resveratrol synthesis, whereas cold storage $\left(4{ }^{\circ} \mathrm{C}\right)$ decreased its concentration. Cold storage apparently inhibited $E$-resveratrol synthesis after harvest, and its concentration slowly decreased. This may be explained by the action of peroxidase enzymes and the subsequent formation of $\varepsilon$-viniferin. In non-UV-C irradiated grapes, the $E$-resveratrol content increased by nearly 14 times when after 3 days of storage at low temperature, the grapes were stored under mild storage conditions.

After 60 days of withering, Raboso Piave and Corvina grape samples showed an evident increase in the content of most stilbenes (Table 2, no. 18) (De Rosso et al. 2016). Raboso Piave had a statistically significant increase in $E$-resveratrol, pallidol, and $E$ - $\varepsilon$ viniferin. In Corvina grape, $E$-resveratrol, piceatannol, $E$-astringin, $E$-piceid, pallidol, pallidol glucoside, $E$ miyabenol $\mathrm{C}$, and $\varepsilon$-viniferin increased significantly. In general, these findings are in agreement with those reported in a study of Aleatico grape withering (Mencarelli et al. 2010).

\section{Combined abiotic pre- and postharvest treatments of grape}

Preharvest red grapes (Syrah Vitis vinifera L.) treated with methyl jasmonate (MEJA) showed a significant increase in $E$-resveratrol and piceatannol contents compared to those in the control sample (piceatannol not detected) (Table 2, no. 19) (Fernández-Marín et al. 2014). The highest concentrations of piceatannol and $E$-resveratrol were found at harvest. Larronde et al. (2003) described an $E$-resveratrol increase (ninefold) in Cabernet sauvignon berries when treated with MEJA vapours 15 days after veraison. However, berries rapidly lose the capacity to respond to MEJA with ripening. Vezzulli et al. (2007) found that MEJA treatment of the Barbera grape variety improved the $E$ resveratrol and $\varepsilon$-viniferin contents in an accumulative manner. Thus, it seems that there is an effect of MEJA on the stilbene concentration in grapes.

Harvested red grapes were treated with UV-C irradiation and stored for 4 days (Table 2, no. 19) (Fernández-Marín et al. 2014). On the 4th day, control red grapes showed a significantly lower $E$-resveratrol content than grapes treated with UV-C, and UV-C samples a lower $E$-resveratrol content than MEJA samples, whereas MEJA-UV-C achieved the highest $E$-resveratrol content. In fact, UV-C irradiation has been described as a stronger stress than MEJA, causing higher $E$-resveratrol induction in grape, but a longer grape storage period was required (Fernández-Marín et al. 2012).

The accumulation of piceatannol, isorhapontigenin, and $\varepsilon$-viniferin were also induced (Table 2, no. 19). At harvest, piceatannol was found in MEJA grapes, but it was absent in control samples. After 2 days of storage, piceatannol was found in every batch, and isorhapontigenin was found in the MEJA and MEJA-UV-C batches in concentrations that were not significantly different. The trend of these stilbenes was similar to that of E-resveratrol. The control batch showed the lowest concentration, in contrast to MEJA-UV-C, which showed the highest concentration after 4 days of storage.

The effectiveness of the chitosan treatment of table grapes (Autumn Black and B36-55 variety) in combination with UV-C irradiation to determine the $E$-resveratrol concentration in grape berry skins was investigated (Table 2, no. 20) (Romanazzi et al. 2006). Grape berries were sprayed in the vineyard with $1 \%$ chitosan and then harvested daily for 5 days. Immediately after harvest, they were inoculated with $B$. cinerea. In cv. Autumn Black and selection B36-55, Eresveratrol was not detected in control berries or berries with a chitosan coating. In berries exposed to UV-C irradiation alone and in berries treated with $1 \%$ chitosan coating and later exposed UV-C, E-resveratrol was found. The berries treated with the combination of chitosan and UV-C irradiation contained approximately 11 times more $E$-resveratrol than the control grapes and 0.2 times more than those only irradiated with UV-C. Z-Resveratrol, E-piceid, and Zpiceid were not detected in any of the samples.

\section{Biotic postharvest treatments of grape}

Infection of the postharvested Palomino fino grapes with Botrytis cinerea led to a domination of piceid over resveratrol (Table 2, no. 21) (Roldán et al. 2003). At the early stage of fungal development, the content of piceid increased more than onefold in fruits over that in the control sample, while the level of resveratrol decreased more than onefold. At more advanced infection stages, the piceid and resveratrol contents 
increased 3.5-fold and 0.4-fold, respectively. According to the literature, resveratrol synthesis occurs in the skin of edible berries (Sotheeswaran and Pasupathy 1993). Thus, an accumulation of resveratrol in the skin after infection by $B$. cinerea could be expected. However, the results showed that even at the early stage of pathogen development, a sharp decrease in the resveratrol content occurred. This could be because before pathogen exposure, resveratrol synthesis is stimulated. The resveratrol already present in the skin is used by the fruit as part of its defence mechanism. This phytoalexin would become a building block of stilbenes such as $\varepsilon$ - and $\delta$-viniferins, which are more toxic to the pathogen. For this reason, the piceid and resveratrol contents decreased. In the second stage of pathogen development, an accumulation of resveratrol occurred until a certain level was reached, and it was maintained during external stress. These results are in accordance with the literature, which showed that in grape berry skins infected by powdery mildew, the resveratrol and piceid isomers were considerably increased and the degree of infection was positively related to their stilbene content (Romero et al. 2001).

Infection with Aspergillus carbonarius at ripening induced in the grapes an approximately onefold increase in $E$-resveratrol and resveratrol dimers, such as $\omega$-viniferin, $E$ - $\varepsilon$-viniferin, caraphenol and $\delta$-viniferin, and resveratrol trimers, such as $\alpha$-viniferin and E-miyabenol C (Table 2, no. 22) (Flamini et al. 2016). However, infection with this pathogen decreased the $E$-piceid and $E$-astringin concentrations by approximately onefold in grapes. The total stilbene content in grapes infected with A. carbonarius increased $60 \%$ over that in uninfected berries. Glycosylation of stilbenes is involved in storage, transport from the cytoplasm to the apoplasm and protection from peroxidative degradation. Subsequent storage in vacuoles may protect plant cells from potentially their toxic effects. In susceptible cultivars, such as Gamay, Gamaret, Pinot Noir and Chasselas, resveratrol was found to be either glycosylated or present in very low concentrations (Pezet et al. 2004). Studies of grapevine varieties resistant to $P$. viticola reported that $E$ resveratrol is probably rapidly oxidized into toxic stilbenes, such as $\varepsilon^{-}$and $\delta$-viniferins, while in susceptible cultivars, it is rapidly glycosylated into less toxic piceid (Pezet et al. 2004). In the case of infected Negro Amaro grape, the content of $E$ resveratrol increased, but the content of glycosylated stilbenes decreased, which confirms the low susceptibility of this cultivar against $A$. carbonarius infection.

In the research conducted by Bavaresco et al. (2003) the influence of fungal infection with $A$. japonicus, A. ochraceus, A. fumigatus and A. carbonarius on the stilbenes content in the grape berries was examined. All tested fungi, except A. fumigatus, significantly increased $E$-resveratrol concentration over the control sample, while $E$-piceid content was not affected. Among tested fungi, only A. ochraceus significantly elicited the grape berries to synthesize piceatannol.

\section{Combined abiotic and biotic postharvest treatments of grape}

In another study, phenyl propanoid metabolism induction by abiotic (UV-C) and biotic elicitors (fungal infection-ochratoxigenic Aspergillus) in undamaged and damaged Napoleon table grapes was investigated (Table 2, no. 23) (Selma et al. 2008). In addition, the effect of the sequence of elicitors on the content of stilbenes and storage time was analysed. The contents of $E$-resveratrol in non-inoculated and inoculated grapes were similar and maintained at the same level during 5 days of storage. Treatment of undamaged or damaged grapes with UV-C and fungal infection increased the content of $E$-resveratrol 6 times ( 7 or 4 times for undamaged and damaged berries, respectively, in the case of infection and subsequent UV-C treatment) compared with that in non-inoculated samples, after 5 days of storage. In addition, the $E$ resveratrol content did not increase in damaged grapes compared to that in undamaged grapes either at day 0 or after storage. These results suggest that $E$-resveratrol was not elicited by ochratoxigenic Aspergillus. However, in another study, it has been shown that $E$ resveratrol accumulates in $A$. carbonarius inoculated grapes at veraison time but not during ripening (Bavaresco et al. 2003). Moreover, E-resveratrol formation decreases from veraison to ripening in berries, and this formation is elicited by biotic (ochratoxigenic Aspergillus) and abiotic (UV-C) factors (Jeandet et al. 1991). However, the induction capacity for $E$-resveratrol was sevenfold, which is higher than the value of 4.4-fold obtained in another study (Cantos et al. 2002). The accumulation of $E$ - 
resveratrol was significantly enhanced by increasing the storage time and by UV-C treatment, as well as by the combination of both treatments.

Inoculation with ochratoxigenic Aspergillus did not specifically elicit the accumulation of $E$-piceid (Table 2, no. 23). However, there was a significant increase in the induction of $E$-piceid during storage, and this induction was particularly faster in damaged berries than in undamaged grapes. Therefore, $E$-piceid induction in UV-C untreated grapes could be a consequence of the total microbial contamination rather than a specific response to ochratoxigenic Aspergillus. On the other hand, in undamaged berries, the $E$-piceid content increase in response to microbial infection was faster in UV-C treated berries than in untreated berries. The $E$-piceid content in damaged grapes after treatment with UV-C and fungal infection increased onefold.

UV-C treatment induced the biosynthesis of $E$ resveratrol as a result of an increased transcription of genes encoding stilbene synthase. Fungal infection induced not only the transcription of stilbene synthase but also the transcription of genes encoding glycosyltransferases, which transform $E$-resveratrol to $E$ piceid.

\section{Conclusions}

Edible berries are a rich source of nutritive and bioactive compounds, including stilbenes, among which E-resveratrol, piceatannol, Z/E-piceid and viniferin are the most common. The extraction techniques applied in stilbene isolation can be classified into two categories: green techniques and conventional techniques. Conventional techniques use a large volume of solvent, especially methanol or ethanol and their aqueous mixtures or mixtures with other solvents. Green techniques are not as common as conventional techniques but allow minimalization of solvent usage.

Among the analysed edible berries, the highest concentration of stilbenes has been found in passion fruit (Passiflora edulis). However, the highest concentration of $E$-resveratrol in the skin has been found in table grapes (Vitis vinifera $\mathrm{L}$.). The quantitative and qualitative composition of stilbenes in edible berries depends on many environmental factors. However, the application of various abiotic and biotic external stimuli may increase the production of stilbenes in grapes. Several different elicitors, as inducers of secondary metabolite stilbenes, were analysed: UV irradiation, visible light, ultrasonication, signalling compounds and fungicides. Among them, UV treatment was the most effective method to enhance stilbene derivative production. The enhancement of the stilbene content depended on the UV wavelength, UV intensity, duration of irradiation and developmental stage of the berry fruit. The STS expression responsible for stilbene production was the highest when the berries were exposed to UV-C irradiation at 2 weeks before veraison. In general, the effect of biotic stressors, such as Aspergillus carbonarius and Botrytis cinerea, was found to be not as efficient as the effect of abiotic elicitors.

Open Access This article is distributed under the terms of the Creative Commons Attribution 4.0 International License (http:// creativecommons.org/licenses/by/4.0/), which permits unrestricted use, distribution, and reproduction in any medium, provided you give appropriate credit to the original author(s) and the source, provide a link to the Creative Commons license, and indicate if changes were made.

\section{References}

Adrian M, Jeandet P, Douillet-Breuil AC, Tesson L, Bessis R (2000) Stilbene content of mature Vitis vinifera berries in response to UV-C elicitation. J Agric Food Chem 48:6103-6105

Ahn SY, Kim SA, Choi SJ et al (2015) Comparison of accumulation of stilbene compounds and stilbene related gene expression in two grape berries irradiated with different light sources. Hortic Environ Biotechnol 56(1):36-43

Artés-Hernández F, Artés F, Tomás-Barberán FA (2003) Quality and enhancement of bioactive phenolics in cv. Napoleon table grapes exposed to different postharvest gaseous treatments. J Agric Food Chem 51(18):5290-5295

Babazadeh A, Taghvimi A, Hamishehkar H, Tabibiazar M (2017) Development of new ultrasonic-solvent assisted method for determination of trans-resveratrol from red grapes: optimization, characterization, and antioxidant activity (ORAC assay). Food Bioscience 20:36-42

Bai Y, Mao QQ, Qin J et al (2010) Resveratrol induces apoptosis and cell cycle arrest of human T24 bladder cancer cells in vitro and inhibits tumor growth in vivo. Cancer Sci 101(2):488-493

Bavaresco L, Fregoni M, Trevisan M et al (2002) The occurrence of the stilbene piceatannol in grapes. Vitis 41(3): 133-136

Bavaresco L, Vezzulli S, Battilani P et al (2003) Effect of ochratoxin A-producing Aspergilli on stilbenic phytoalexin synthesis in grapes. J Agric Food Chem 51(21):6151-6157 
Burin VM, Ferreira-Lima NE, Panceri CP, Bordignon-Luiz MT (2014) Bioactive compounds and antioxidant activity of Vitis vinifera and Vitis labrusca grapes: evaluation of different extraction methods. Microchemical J 114:155-163

Cantos E, García-Viguera C, de Pascual-Teresa S et al (2000) Effect of postharvest ultraviolet irradiation on resveratrol and other phenolics of cv. Napoleon table grapes. J Agric Food Chem 48(10):4606-4612

Cantos E, Espín JC, Tomás-Barberán FA (2001) Postharvest induction modeling method using UV irradiation pulses for obtaining resveratrol-enriched table grapes: a new "functional" fruit? J Agric Food Chem 49(10):5052-5058

Cantos E, Espín JC, Tomás-Barberán FA (2002) Postharvest stilbene-enrichment of red and white table grape varieties using UV-C irradiation pulses. J Agric Food Chem 50(22):6322-6329

Castrejon ADR, Eichholz I, Rohn S et al (2008) Phenolic profile and antioxidant activity of highbush blueberry (Vaccinium corymbosum L.) during fruit maturation and ripening. Food Chem 109:564-572

Cayuela JA, Vázquez A, Pérez AG et al (2010) Control of table grapes postharvest decay by ozone treatment and resveratrol induction. Food Sci Technol Int 15(5):495-502

Cho YJ, Hong JY, Chun HS, Lee SK, Min HY (2006) Ultrasonication-assisted extraction of resveratrol fromgrapes. J Food Eng 77:725-730

Cho YJ, Kim N, Kim CT, Maeng JS, Pyee J (2012) Quantitative evaluation of resveratrol enrichment induced by UV stimulus in harvested grapes. Food Sci Biotechnol 21(2):597-601

Chong J, Poutaraud A, Hugueney P (2009) Metabolism and roles of stilbenes in plants. Plant Sci 177:143-155

Coutos-Thévenot P, Poinssot B, Bonomelli A et al (2001) In vitro tolerance to Botrytis cinerea of grapevine 41B rootstock in transgenic plants expressing the stilbene synthase Vst 1 gene under the control of a pathogen-inducible PR10 promoter. J Exp Bot 52(358):901-910

Crupi P, Pichierri A, Basile T et al (2013) Postharvest stilbenes and flavonoids enrichment of table grape cv Redglobe (Vitis vinifera L.) as affected by interactive UV-C exposure and storage conditions. Food Chem 141(2):802-808

De Rosso M, Soligo S, Panighel A et al (2016) Changes in grape polyphenols ( $V$. vinifera L.) as a consequence of postharvest withering by high-resolution mass spectrometry: raboso Piave versus Corvina. J Mass Spectrom 51(9):750-760. https://doi.org/10.1002/jms.3835

Dixon RA, Paiva NL (1995) Stress-induced phenylpropanoid metabolism. Plant Cell 7:1085-1097

Douillet-Breuil AC, Jeandet P, Adrian M, Bessis R (1999) Changes in the phytoalexin content of various Vitis spp. in response to UV-C elicitation. J Agric Food Chem 47:4456-4461

Ehala S, Vaher M, Kaljurand M (2005) Characterization of phenolic profiles of Northern European berries by capillary electrophoresis and determination of their antioxidant activity. J Agric Food Chem 53(16):6484-6490

Fernández-Marín MI, Guerrero RF, García-Parrilla MC et al (2012) Isorhapontigenin: a novel bioactive stilbene from wine grapes. Food Chem 135:1353-1359

Fernández-Marín MI, Guerrero RF, García-Parrilla MC et al (2013) Terroir and variety: two key factors for obtaining stilbene-enriched grapes. J Food Compos Anal 31(2):191-198

Fernández-Marín MI, Puertas B, Guerrero RF et al (2014) Preharvest methyl jasmonate and postharvest UVC treatments: increasing stilbenes in wine. $J$ Food Sci 79(3):C310-C317. https://doi.org/10.1111/1750-3841. 12368

Flamini R, de Rosso M, de Marchi F et al (2013) An innovative approach to grape metabolomics: stilbene profiling by suspect screening analysis. Metabolomics 9(6):1243-1253

Flamini R, Zanzotto A, de Rosso M et al (2016) Stilbene oligomer phytoalexins in grape as a response to Aspergillus carbonarius infection. Physiol Mol Plant Pathol 93:112-118

Freitas PM, López-Gálvez F, Tudela JA et al (2015) Postharvest treatment of table grapes with ultraviolet- $\mathrm{C}$ and chitosan coating preserves quality and increases stilbene content. Postharvest Biol Technol 105:51-57

Gonzalez Ureña A, Orea JM, Montero C et al (2003) Improving postharvest resistance in fruits by external application of trans-resveratrol. J Agric Food Chem 51(1):82-89

González-Barrio R, Beltran D, Cantos E, Gil MI, Espin JC, Tomas-Barberán FA (2006) Comparison of ozone and UV$\mathrm{C}$ treatments on the postharvest stilbenoid monomer, dimer, and trimer induction in var. 'Superior' white table grapes. J Agric Food Chem 54:4222-4228

Guerrero RF, Puertas B, Fernández MI et al (2010a) Induction of stilbenes in grapes by UV-C: comparison of different subspecies of Vitis. Innov Food Sci Emerg Technol 11(1):231-238

Guerrero RF, Puertas B, Fernández MI et al (2010b) UVCtreated skin-contact effect on both white wine quality and resveratrol content. Food Res Int 43(8):2179-2185

Guerrero RF, Cantos-Villar E, Fernández-Marín MI et al (2015) Optimising UV-C preharvest light for stilbene synthesis stimulation in table grape: applications. Innov Food Sci Emerg Technol 29:222-229

Guerrero RF, Cantos-Villar E, Puertas B et al (2016) Daily preharvest UV-C light maintains the high stilbenoid concentration in grapes. $\mathrm{J}$ Agric Food Chem 64(25):5139-5147

Hasan MM, Bae H (2017) An overview of stress-induced resveratrol synthesis in grapes: perspectives for resveratrol-enriched grape products. Molecules 22(294):1-18

Hasan MM, Baek KH (2013) Induction of resveratrol biosynthesis in grape skin and leaves by ultrasonication treatment. Korean J Hortic Sci Technol 31:496-502

He S, Jiang L, Wu B et al (2009a) Chunganenol: an unusual antioxidative resveratrol hexamer from Vitis chunganensis. J Org Chem 74(20):7966-7969

He S, Lu Y, Jiang L et al (2009b) Preparative isolation and purification of antioxidative stilbene oligomers from Vitis chunganeniss using high-speed counter-current chromatography in stepwise elution mode. J Sep Sci 32(14):2339-2345

Holl J, Vannozzi A, Czemmel S et al (2013) The R2R3-MYB transcription factors MYB14 and MYB15 regulate stilbene biosynthesis in Vitis vinifera. Plant Cell 25:4135-4149

Jeandet P, Bessis R, Gautheron B (1991) The production of resveratrol $\left(3,5,4^{\prime}\right.$-trihydroxystilbene) by grape berries in different developmental stages. Am J Enol Vitic 42:41-44 
Jensen JS, Wertz CF, O’Neill VA (2010) Preformulation stability of trans-resveratrol and trans-resveratrol glucoside (piceid). J Agric Food Chem 58(3):1685-1690. https://doi. org/10.1021/jf903009f

Jiang L, He S, Sun C et al (2012) Selective ${ }^{1} \mathrm{O}_{2}$ quenchers, oligostilbenes, from Vitis wilsonae: structural identification and biogenetic relationship. Phytochemistry 77:294-303

Jiménez Sánchez JB, Crespo Corral E, Orea MJ et al (2007) Elicitation of trans-resveratrol by laser resonant irradiation of table grapes. Appl Phys B 87(3):559-563. https://doi. org/10.1007/s00340-007-2591-0

Jiménez JB, Orea JM, Gonzalez Ureña A, Escribano P, López de al Osa P, Guadarrama A (2007) Short anoxic treatments to enhance trans-resveratrol content in grapes and wine. Eur Food Res Technol 224:373-378

Jimenez-Garcia SN, Guevara-Gonzalez RG, Miranda-Lopez R et al (2013) Functional properties and quality characteristics of bioactive compounds in berries: biochemistry, biotechnology, and genomics. Food Res Int 54:1195-1207

Kasiotis KM, Pratsinis H, Kletsas D et al (2013) Resveratrol and related stilbenes: their anti-aging and anti-angiogenic properties. Food Chem Toxicol 61:112-120

Kawakami S, Kinoshita Y, Maruki-Uchida H et al (2014) Piceatannol and its metabolite, isorhapontigenin, induce SIRT1 expression in THP-1 human monocytic cell line. Nutrients 6(11):4794-4804

Kiselev KV, Aleynova OA, Grigorchuk VP et al (2017) Stilbene accumulation and expression of stilbene biosynthesis pathway genes in wild grapevine Vitis amurensis Rupr. Planta 245(1):151-159

Langcake P, Pryce RJ (1977) The production of resveratrol and the viniferins by grapevines in response to ultraviolet irradiation. Phytochem 16:1193-1196

Larronde F, Gaudillere JP, Krisa S et al (2003) Air-borne methyl jasmonate induces stilbene accumulation in leaves and berries of grapevine plants. Am J Enol Vitic 54:63-66

Lin M, Yao CS (2006) Natural oligostilbenes. Stud Nat Prod Chem 33:601-644

Liu W, Liu CY, Yang CX et al (2010) Effect of grape genotype and tissue type on callus growth and production of resveratrols and their piceids after UV-C irradiation. Food Chem 122:475-481

Mencarelli F, Bellincontro A, Nicoletti I et al (2010) Chemical and biochemical change of healthy phenolic fractions in winegrape by means of postharvest dehydration. J Agric Food Chem 58(13):7557-7564

Morales M, Bru R, Garcia-Carmona F et al (1998) Effect of dimethyl- $\beta$-cyclodextrins on resveratrol metabolism in Gamay grapevine cell cultures before and after inoculation with Xylophilus ampelinus. Plant Cell Tissue Organ Cult 53(3):179-187

Moriartry JM, Harmon R, Weston LA et al (2001) Resveratrol content of two Californian table grape cultivars. Vitis 40(1):43-44

Može S, Polak T, Gasperlin L et al (2011) Phenolics in Slovenian bilberries (Vaccinium myrtillus L.) and blueberries (Vaccinium corymbosum L.). J Agric Food Chem 59(13):6998-7004
Nguyen TNA, Dao TT, Tung BT et al (2011) Influenza A (H1N1) neuraminidase inhibitors from Vitis amurensis. Food Chem 124(2):437-443

Nile SH, Park SW (2014) Edible berries: bioactive compounds and their effect on human health. Nutrition 30:134-144

Palou L, Crisosto CH, Smilanick JL, Adaskaveg JE, Zoffoli JP (2002) Effects of continuous 0.3 ppm ozone exposure on decay development and physiological responses of peaches and table grapes in cold storage. Postharvest Biol Technol 24:39-48

Petit AN, Baillieul F, Vaillant-Gaveau N, Jacquens L, Conreux A, Jeandet P, Clement C, Fontaine F (2009) Low responsiveness of grapevine flowers and berries at fruit set to UVC irradiation. J Exp Bot 60:1155-1162

Pezet R, Gindro K, Viret O et al (2004) Glycosylation and oxidative dimerization of resveratrol are respectively associated to sensitivity and resistance of grapevine cultivars to downy mildew. Physiol Mol Plant Pathol 65:297-303

Pilati S, Perazzolli M, Malossini A et al (2007) Genome-wide transcriptional analysis of grapevine berry ripening reveals a set of genes similarly modulated during three seasons and the occurrence of an oxidative burst at veraison. BMC Genomics 8:428. https://doi.org/10.1186/1471-2164-8-428

Prokop J, Abrman P, Seligson AL et al (2006) Resveratrol and its glycon piceid are stable polyphenols. J Med Food 9(1):11-14

Ragab AS, Van Fleet J, Jankowski B et al (2006) Detection and quantitation of resveratrol in tomato fruit (Lycopersicon esculentum Mill.). J Agric Food Chem 54(19):7175-7179

Ramprasath VR, Jones PJH (2010) Anti-atherogenic effects of resveratrol. Eur J Clin Nutr 64(7):660-668

Rimando AM, Cody R (2005) Determination of stilbenes in blueberries. LCGC N Am 23(11):1192-1200

Roldán A, Palacios V, Caro I et al (2003) Resveratrol content of Palomino fino grapes: influence of vintage and fungal infection. J Agric Food Chem 51(5):1464-1468

Romanazzi G, Mlikota-Gabler F, Smilanick JL (2006) Preharvest chitosan and postharvest UV irradiation treatments suppress gray mold of table grapes. Plant Dis 90(4):445-450

Romero AI, Lamuela-Raventós RM, Adrés-Lacueva C et al (2001) Method for the quantitative extraction of resveratrol and piceid isomers in grape berry skins. Effect of powdery mildew on the stilbene content. J Agric Food Chem 49(1):210-215

Ros Barcelo A, Pomar F, Lopez-Serrano M et al (2003) Peroxidase: a multifunctional enzyme in grapevines. Funct Plant Biol 30(6):577-591

Samoticha J, Wojdyło A, Golis T (2017) Phenolic composition, physicochemical properties and antioxidant activity of interspecific hybrids of grapes growing in Poland. Food Chem 215:263-273

Sarig P, Zahavi T, Zutkhi Y, Yannai S, Lisker N, Ben-Arie R (1996) Ozone for control of post-harvest decay of table grapes caused by Rhizopus stolonifer. Physiol Mol Plant Pathol 48:403-415

Seeram NP (2012) Emerging research supporting the positive effects of berries on human health and disease prevention. J Agric Food Chem 60(23):5685-5686 
Selma MV, Freitas PM, Almela L et al (2008) Ultraviolet-C and induced stilbenes control ochratoxigenic Aspergillus in grapes. J Agric Food Chem 56(21):9990-9996

Sotheeswaran S, Pasupathy V (1993) Distribution of resveratrol oligomers in plants. Phytochem 32:1083-1092

Sun B, Ribes AM, Conceicao Leandro M et al (2006) Stilbenes: quantitative extraction from grape skins, contribution of grape solids to wine and variation during wine maturation. Anal Chim Acta 563:382-390

Suzuki M, Nakabayashi R, Ogata Y et al (2015) Multiomics in grape berry skin revealed specific induction of the stilbene synthetic pathway by ultraviolet-C irradiation. Plant Physiol 168(1):47-59

Tsai HY, Ho CT, Chen YK (2017) Biological actions and molecular effects of resveratrol, pterostilbene and 3'-hydroxypterostilbene. J Food Drug Anal 25(1):134-147

Versari A, Parpinello GP, Tornielli GB, Ferrarini R, Giulivo C (2001) Stilbene compounds and stilbene synthase expression during ripening, wilting, and UV treatment in grape cv. Corvina. J Agric Food Chem 49:5531-5536

Vezzulli S, Civardi S, Ferrari F et al (2007) Methyl jasmonate treatment as a trigger of resveratrol synthesis in cultivated grapevine. Am J Enol Vitic 58:530-533

Vilanova M, Rodríguez I, Canosa P et al (2015) Variability in chemical composition of Vitis vinifera cv Mencía from different geographic areas and vintages in Ribeira Sacra (NW Spain). Food Chem 169:187-196

Viñas P, Campillo N, Martínez-Castillo N et al (2009) Solidphase microextraction on-fiber derivatization for the analysis of some polyphenols in wine and grapes using gas chromatography-mass spectrometry. J Chromatogr A 1216(9):1279-1284

Viñas P, Martínez-Castillo N, Campillo N et al (2011) Directly suspended droplet microextraction with in injection-port derivatization coupled to gas chromatography-mass spectrometry for the analysis of polyphenols in herbal infusions, fruits and functional foods. J Chromatogr A 1218(5):639-646

Vincenzi S, Tomasi D, Gaiotti F et al (2013) Comparative study of the resveratrol content of twenty-one Italian red grape varieties. S Afr J Enol Vitic 34(1):30-35

Vrhovsek U, Masuero D, Gasperotti M et al (2012) A versatile targeted metabolomics Method for the rapid quantification of multiple classes of phenolics in fruits and beverages. J Agric Food Chem 60(36):8831-8840

Wang W, Tang K, Yang HR et al (2010) Distribution of resveratrol and stilbene synthase in young grape plants (Vitis vinifera L. cv. Cabernet Sauvignon) and the effect of UV-C on its accumulation. Plant Physiol Biochem 48:142-152

Wang L, Ma L, Xi HF et al (2013) Individual and combined effects of $\mathrm{CaCl}_{2}$ and UV-C on the biosynthesis of resveratrols in grape leaves and berry skins. J Agric Food Chem 61(29):7135-7141

Wang JF, Ma L, Xi HF et al (2015) Resveratrol synthesis under natural conditions and after UV-C irradiation in berry skin is associated with berry development stages in 'Beihong' (V. vinifera $\times V$. amurensis). Food Chem 168:430-438

Yin X, Singer SD, Qiao H, Liu Y, Jiao C, Wang H, Li Z, Fei Z, Wang Y, Fan C et al (2016) Insights into the mechanisms underlying ultraviolet-C induced resveratrol metabolism in grapevine (V. amurensis Rupr.) cv. "Tonghua-3". Front Plant Sci 7:1-16

Zhang F, Liu J, Shi JS (2010) Anti-inflammatory activities of resveratrol in the brain: role of resveratrol in microglial activation. Eur J Pharmacol 636:1-7

Zhao Y (ed) (2007) Berry fruit value-added products for health promotion. CRC Press, Boca Raton 